Research paper

\title{
Numerical modeling for strain rate effect and size effect of ice under uniaxial tension and compression
}

\author{
Jian-Ping Zhanga, Dong Zhou ${ }^{\mathrm{b}, *}$ \\ a Civil Aviation Flight University of China, Guanghan, Sichuan, 618300, China \\ ${ }^{\mathrm{b}}$ Institute of Mechanics, Chinese Academy of Sciences, Beijing, 100190, China
}

\section{A R T I C L E I N F O}

\section{Article history:}

Received 18 November 2019

Revised 6 September 2020

Accepted 5 November 2020

Available online 11 November 2020

\section{Keywords:}

PSM

Brittle failure

Ice

Strain rate effect

Size effect

\begin{abstract}
A B S T R A C T
In order to study the strain rate effect and size effect during the brittle failure process of ice under uniaxial tension and compression with numerical means, particle-subdomain method (PSM) is introduced. PSM is a continuum-discontinuum coupled method with time-dependent explicit algorithm of dynamic relaxation, which has combined the advantages of particle-in-cell method, finite element method and discrete element method. The governing equation of this method is deduced from Lagrange equation, which is a unification of expression for continuous deformation, fracture and failure. The complex failure behaviors of brittle ice under uniaxial tension and compression in laboratory scale are simulated. The progressive failure process with fracture evolution can be well described with this method. The results show that failure mode and the strength of ice material are affected by loading rate, boundary conditions and the size of the specimen. The strain rate effect and size effect with transition property are naturally obtained and can be explained with internal fracture evolution process within the material.
\end{abstract}

(C) 2020 Published by Elsevier B.V.

\section{Introduction}

Ice is a quasi-brittle material with obvious characteristics of heterogeneity and discontinuity. Especially for sea ice, which consists of solid ice, brine and gas, would exhibit different grain structures and grain size that depending upon the temperature, salts and other environmental conditions [42]. The changeable meteorological condition and disturbance of waves could result in chaotic microstructures of ice in nature. There is a very large variation in the grain size and shape even over small distances. Meanwhile, lots of voids and discontinuous interfaces exist within the ice material. Thus, the mechanical property and behavior of ice could be quite complex. Failure process of ice should accompany with deformation, crack growth, and even movement of fragments.

There have been a large number of tests performed to research the mechanical properties and failure process of ice. Tensile and compressive failure were the most concerned failure modes in the lab, and lots of tests have been conducted to measure the tensile strength [20,25,34,35] and compressive strength $[19,27,40,41]$. The strength of ice is affected by the loading rate and the size of the grain relative to the specimen, which is called strain rate effect and size effect respectively $[6,21,32,38,45,47]$. At low loading rate, i.e. lower than $10^{-6}-10^{-5} \mathrm{~s}^{-1}$, the ice material is relatively ductile. The strength increases with the increase of the strain rate, which exhibits little dependence upon the grain size [5,36]. However, at higher loading rate, i.e. above $10^{-4}-10^{-3} \mathrm{~s}^{-1}$, ice exhibits brittle behavior, and the strength decreases with the increase

\footnotetext{
* Corresponding author.

E-mail addresses: zdstar@live.com, zhoudong@imech.ac.cn (D. Zhou).
} 
of the strain rate and the grain size. The brittle failure envelope of ice is different depending upon the loading condition. Uniaxial compression without confinement will result in longitudinal splitting, while compression with confinement would lead to inclined shear fault [37].

In order to describe the mechanical behavior of ice precisely, numerical method must be capable of simulating the whole process of failure. Not only continuous deformation, but also fracture and interactions at the cracks within the ice material should be calculated properly. And the latter usually plays the dominant role in the failure process. Finite element method and discrete element method are the most wildly applied computational frameworks to simulate the brittle failure of ice $[31,39,43,48,49]$. However, study on numerical modeling for ice failure in laboratory scale is scarce. More advanced computational algorithm is required to obtain more realistic failure behavior.

The objective of this paper is to simulate and investigate the brittle failure properties of ice under tension and compression in laboratory scale with a new numerical method called particle-subdomain method (PSM). In Section 2, the basic concept of PSM with systematic expressions is introduced and numerical examples for validation are demonstrated. In Section 3, tensile failure and compressive failure of ice are modeled and validated respectively, strain rate effect and size effect are discussed. Major conclusions are drawn in Section 4.

\section{Theory of particle-subdomain method (PSM)}

\subsection{Basic concept}

PSM is a new type of continuum-discontinuum coupled method, which is based on time-dependent explicit iteration with dynamic relaxation (RD, see [9,10,12,14,29,44]). PSM has combined the advantages of particle-in-cell method (PIC, see e.g. [1-3,15]; Sulsky, 1994,1995; Bardenhagen and Kober, 2004), finite element method (FEM, see e.g. Zienkiewicz and Taylor, 1977; Johnson, 1987) and discrete element method (DEM, see e.g. Cundall and Strack,1979; Cundall and Hart, 1992), which can be widely used in nonlinear, dynamic and fracture problems. It's a mixture of particle system and subdomain system composed of elements. At the very beginning, the initial mesh is divided to establish the computational system. Particles are formed by the nodes. Each particle will get a subdomain composed of all the associate elements, as shown in Fig. 1 . The subdomains can be divided into new ones with new mass and boundaries by the crack surface, when exceeding the strength. The newborn crack surface and boundaries will be detected and calculated with contact and friction law during the computational procedure.

\subsection{Governing equations}

The equation of motion for a particle and associate subdomain in PSM is derived from the Lagrange equation, which is a unification of expression for the continuous system and the discrete system. The first step is to establish the energy function and determine the non-conservative forces for the system.

Kinetic energy $\Pi_{\mathrm{m}}$ of the $N$ th particle can be written as

$$
\Pi_{\mathrm{m}}=\frac{1}{2} \int_{V} \rho \dot{u}_{i}^{2} d V
$$

Where, the roman subscript $\mathrm{m}$ is a text identifier that represents mass, $\rho$ is the density, $i=1,2,3, u_{i}$ is the displacement of the $N$ th particle, $V$ is the volume of the subdomain of the $N$ th particle, $\dot{u}_{i}$ represents the velocity, the first-order derivative of $u_{i}$ versus time.

Potential energy of the Nth particle consists of the strain energy of the subdomain and the work of the conservative force.

The strain energy of the subdomain is

$$
\Pi_{\varepsilon}=\int_{V} \frac{1}{2} \sigma_{i j} \varepsilon_{i j} d V
$$

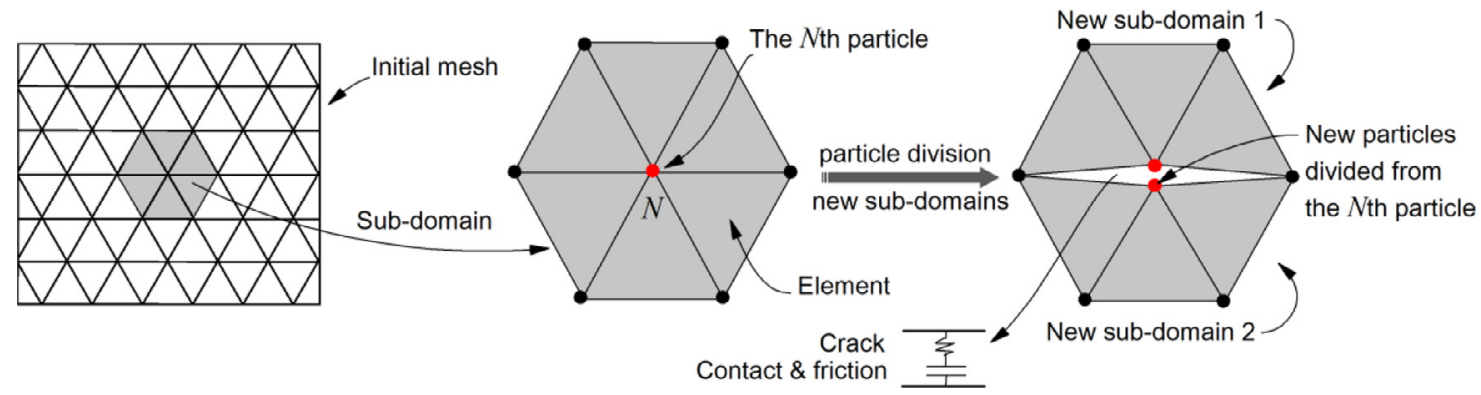

Fig. 1. Basic concept of PSM. The system is composed of particles and subdomains assembled by associate elements. Particles and subdomains can be divided into new ones when cracks initiate. Interactions at the boundaries and cracks are described with contact and friction. 
where, the roman subscript $\varepsilon$ is a text identifier that represents strain, $\sigma_{i j}$ and $\varepsilon_{i j}$ are the stress tensor and strain tensor respectively, $i=1,2,3, j=1,2,3, V$ represents the volume of the subdomain.

The work of conservative force on the $N$ th particle is the work of the body force, which can be expressed as

$$
\Pi_{\mathrm{f}}=-\int_{V} f_{i} u_{i} d V
$$

Where, the roman subscript $\mathrm{f}$ is a text identifier that represents force, $f_{i}$ represents the body force of the $N$ th node.

Non-conservative forces of the Nth particle include the damping force and the external boundary force, that should be written as

$$
\begin{aligned}
& Q_{\mu}=-\int_{V} \mu \dot{u}_{i} d V \\
& Q_{\overline{\mathrm{T}}}=\int_{S} \bar{T}_{i} d S
\end{aligned}
$$

Where $\mu$ is the damping coefficient, $\bar{T}_{i}$ is the external surface force on boundary of the subdomain.

The Lagrange equation is written as

$$
\frac{\partial}{\partial t}\left(\frac{\partial L}{\partial \dot{u}_{i}}\right)-\frac{\partial L}{\partial u_{i}}=Q_{i}
$$

Where $L$ is called the Lagrange function expressed as

$$
L=\Pi_{\mathrm{m}}-\Pi_{\varepsilon}-\Pi_{\mathrm{f}}
$$

And $Q_{i}$ is the non-conservative forces of the system, which is expressed as

$$
Q_{i}=Q_{\mu}+Q_{\bar{T}}
$$

The Lagrange equation needs to take partial derivative with respect to $\dot{u}_{i}$ and $u_{i}$. The energy function should be written as the function of the velocity and the displacement. So the transformation for the expression of strain energy needs to be done.

Eq. (2) can be expressed as the function of strain

$$
\Pi_{\varepsilon}=\int_{V} \frac{1}{2} D_{k l m n} \varepsilon_{k l} \varepsilon_{m n} \mathrm{~d} V
$$

Where $D_{k l m n}$ is the tensor of the elastic constant.

The partial derivative with respect to $u_{i}$ for Eq. (9) is written as

$$
\begin{aligned}
\frac{\partial \Pi_{\varepsilon}}{\partial u_{i}} & =\int_{V}\left(\frac{1}{2} D_{k l m n} \frac{\partial \varepsilon_{k l}}{\partial u_{i}} \varepsilon_{m n}+\frac{1}{2} D_{k l m n} \varepsilon_{k l} \frac{\partial \varepsilon_{m n}}{\partial u_{i}}\right) \mathrm{d} V \\
& =\int_{V}\left(\frac{1}{2} \sigma_{k l} \frac{\partial \varepsilon_{k l}}{\partial u_{i}}+\frac{1}{2} \sigma_{m n} \frac{\partial \varepsilon_{m n}}{\partial u_{i}}\right) \mathrm{d} V \\
& =\int_{V} \sigma_{k l} \frac{\partial \varepsilon_{k l}}{\partial u_{i}} \mathrm{~d} V
\end{aligned}
$$

Where the strain tensor $\varepsilon_{k l}$ can be written as

$$
\varepsilon_{k l}=\frac{1}{2}\left(u_{k, l}+u_{l, k}\right)
$$

Considering the symmetry of the stress tensor $\sigma_{k l}=\sigma_{l k}$, Eq. (10) can be further expressed as

$$
\frac{\partial \Pi_{\varepsilon}}{\partial u_{i}}=\int_{V} \sigma_{k l} \frac{\partial u_{k, l}}{\partial u_{i}} \mathrm{~d} V
$$

According to the formula of integration by parts and Gauss formula, Eq. (12) can be written as

$$
\begin{aligned}
\frac{\partial \Pi_{\varepsilon}}{\partial u_{i}} & =\frac{\partial}{\partial u_{i}} \int_{V} \sigma_{k l} u_{k, l} d V=\frac{\partial}{\partial u_{i}}\left(\int_{V}\left(\sigma_{k l} u_{k}\right)_{, l} d V-\int_{V} \sigma_{k l, l} u_{k} d V\right) \\
& =\frac{\partial}{\partial u_{i}}\left(\int_{S} \sigma_{k l} u_{k} n_{l} d S-\int_{V} \sigma_{k l, l} u_{k} d V\right)=\int_{S} \sigma_{k l} \delta_{i k} n_{l} d S-\int_{V} \sigma_{k l, l} \delta_{i k} d V \\
& =\int_{S} \sigma_{i l} n_{l} d S-\int_{V} \sigma_{i l, l} d V
\end{aligned}
$$

It is equivalent to

$$
\frac{\partial \Pi_{\varepsilon}}{\partial u_{i}}=\int_{S} \sigma_{i j} n_{j} \mathrm{~d} S-\int_{V} \sigma_{i j, j} \mathrm{~d} V
$$

Where $n_{j}$ is the direction cosine. 


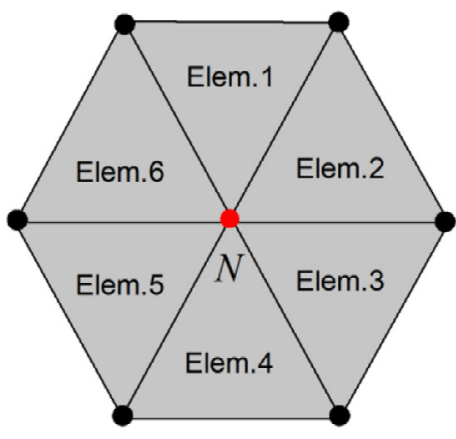

(n)

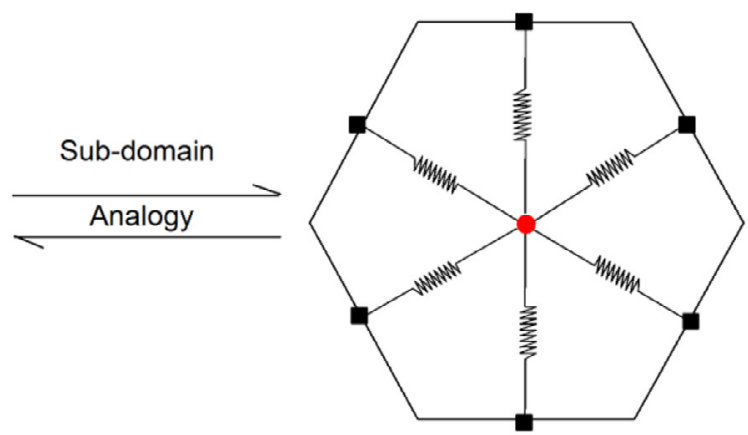

(h)

Fig. 2. The structure of a subdomain and an analogy with a spring system. (a) The structure of a subdomain composed of the associate elements; (b) A spring system used as an analogy to explain the relationship between the subdomain and the central particle. Deformation of the subdomain will produce potential energy, just like energy storage in a spring. Meanwhile, the deformation will impel or constrain the movement of the particle.

With the energy functions, expressions of the non-conservative force and Eq. (14), one could obtain the following equation from the Lagrange equation

$$
\int_{V}\left(\sigma_{i j, j}+f_{i}-\rho \ddot{u}_{i}-\mu \dot{u}_{i}\right) d V+\int_{S}\left(\bar{T}_{i}-\sigma_{i j} n_{j}\right) d S=0
$$

In the problems of dynamic equilibrium, the boundary condition satisfies

$$
\sigma_{i j} n_{j}=\bar{T}_{i}
$$

Then, the differential equation of motion can be derived and written as

$$
\sigma_{i j, j}+f_{i}-\rho \ddot{u}_{i}-\mu \dot{u}_{i}=0
$$

Eqs. (16) and (17) have proved the correctness and validity of the energy function and the non-conservative force system established above.

The subdomain of each particle is composed of all the associate elements, as shown in Fig. 2. The deformation of the subdomain will produce potential energy, and will impel or constrain the movement of the particle. A simplified spring system is shown in Fig. 2 (b) as an analogy, which demonstrates that the movement of the particle is restricted by the associate springs. To determine the force of the subdomain to the particle, one just need to calculate the nodal forces generated by the associate elements, and nodal forces of an element can be easily calculated through finite element method, finite volume method or spring element method, etc. In this work, Finite element method will be used to calculate the nodal force.

According to Fig. 2, Eq. (2) can also be written as

$$
\Pi_{\varepsilon}=\int_{V} \frac{1}{2} \sigma_{i j} \varepsilon_{i j} d V=\sum_{k=1}^{n} \int_{V_{k}} \frac{1}{2} \sigma_{i j} \varepsilon_{i j} d V_{k}
$$

Where $n$ is the number of the elements in a subdomain, $V_{k}$ is the volume of the subdomain from the $k$ th associate element. Thus, force caused by the deformation of the subdomain can be expressed as

$$
F_{i}=\frac{\partial \Pi_{\varepsilon}}{\partial u_{i}}=\sum_{k=1}^{n} \frac{\partial \Pi_{\varepsilon}^{k}}{\partial u_{i}}=\sum_{k=1}^{n} F_{i}^{k}
$$

Where $\Pi_{\varepsilon}^{k}$ is the strain energy of the $k$ th associate element, $n$ is the total number of associate elements, $F_{i}^{k}$ is the local nodal force caused by the deformation of the $k$ th element, which can be calculated by

$$
F_{i}^{k}=K_{i j}^{k} u_{j}^{k}
$$

Where $K_{i j}^{k}$ is the component of the stiffness matrix of the $k$ th element, $u_{j}^{k}$ is the nodal displacements of the $k$ th element.

With Eqs. (1), (3) to (5), and (18) to (20), the equation of motion the Nth particle obtained from Lagrange Eq. (6) can be written as

$$
m \ddot{u}_{i}+c \dot{u}_{i}+F_{i}^{\varepsilon}=F_{i}^{\mathrm{f}}+F_{i}^{\mathrm{T}}
$$

Where $m$ is the mass of the $N$ th particle, $c$ is the damping coefficient, $F_{i}^{\varepsilon}$ is the internal force produced by the associate elements of the $N$ th particle, $F_{i}^{\mathrm{f}}$ is the body force, and $F_{i}^{\mathrm{T}}$ is the external force.

Eq. (21) is the governing equation for each particle and subdomain in PSM. Dynamic relaxation method will be used to iterate over all the particles and subdomains to obtain the whole field resolution within any time step. 


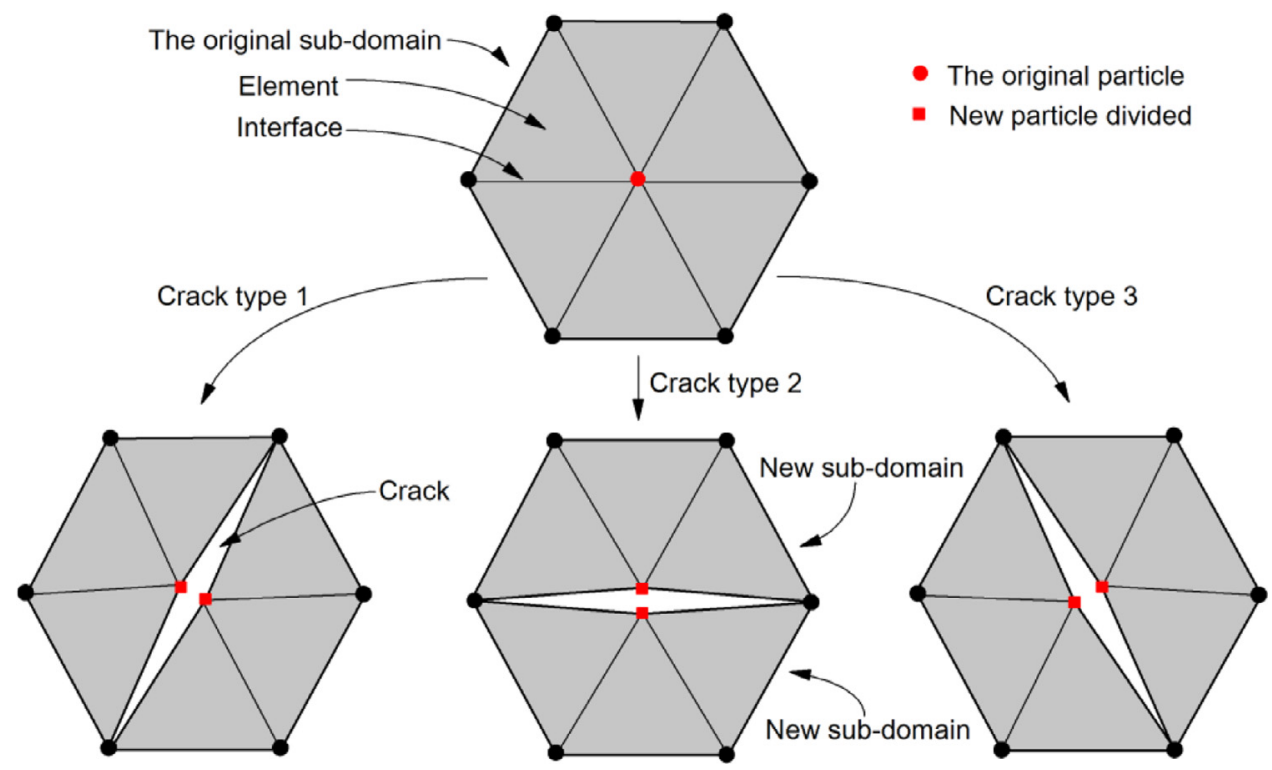

Fig. 3. New particles and new subdomains after breakage. When exceeding the strength, the original particles and subdomain will be divided into new ones along the interfaces of elements according to the stress state.

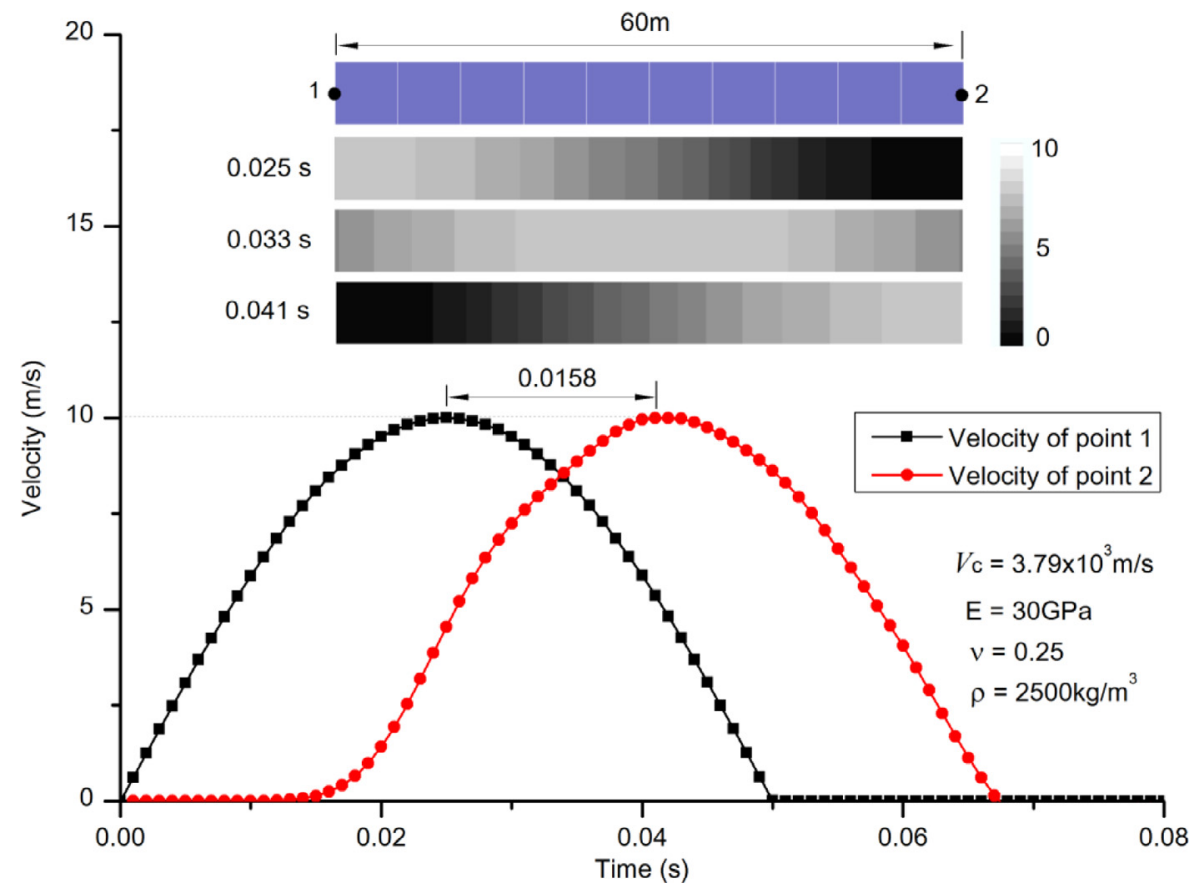

Fig. 4. Wave propagation in a beam. Velocity of a half sinewave is applied to the left end of a free beam with the amplitude of $10 \mathrm{~m} / \mathrm{s}$ and the period of $0.1 \mathrm{~s}$. The wave propagates from the left to the right end of the beam during the calculation. The model and velocity at different time are demonstrated in the Fig.. Wave velocity calculated by PSM agrees with the theoretical value.

\subsection{The damping coefficient}

The damping coefficient is a critical parameter to describe the energy dissipation during the deformation and progressive failure process, which will influence the wave propagation, vibration and the breaking process of the material. So, it's necessary to determine the proper damping coefficient of the material in a simulation.

The governing equation of a node can be written as

$$
m \ddot{u}_{i}+c \dot{u}_{i}+F_{i}^{\varepsilon}=F
$$




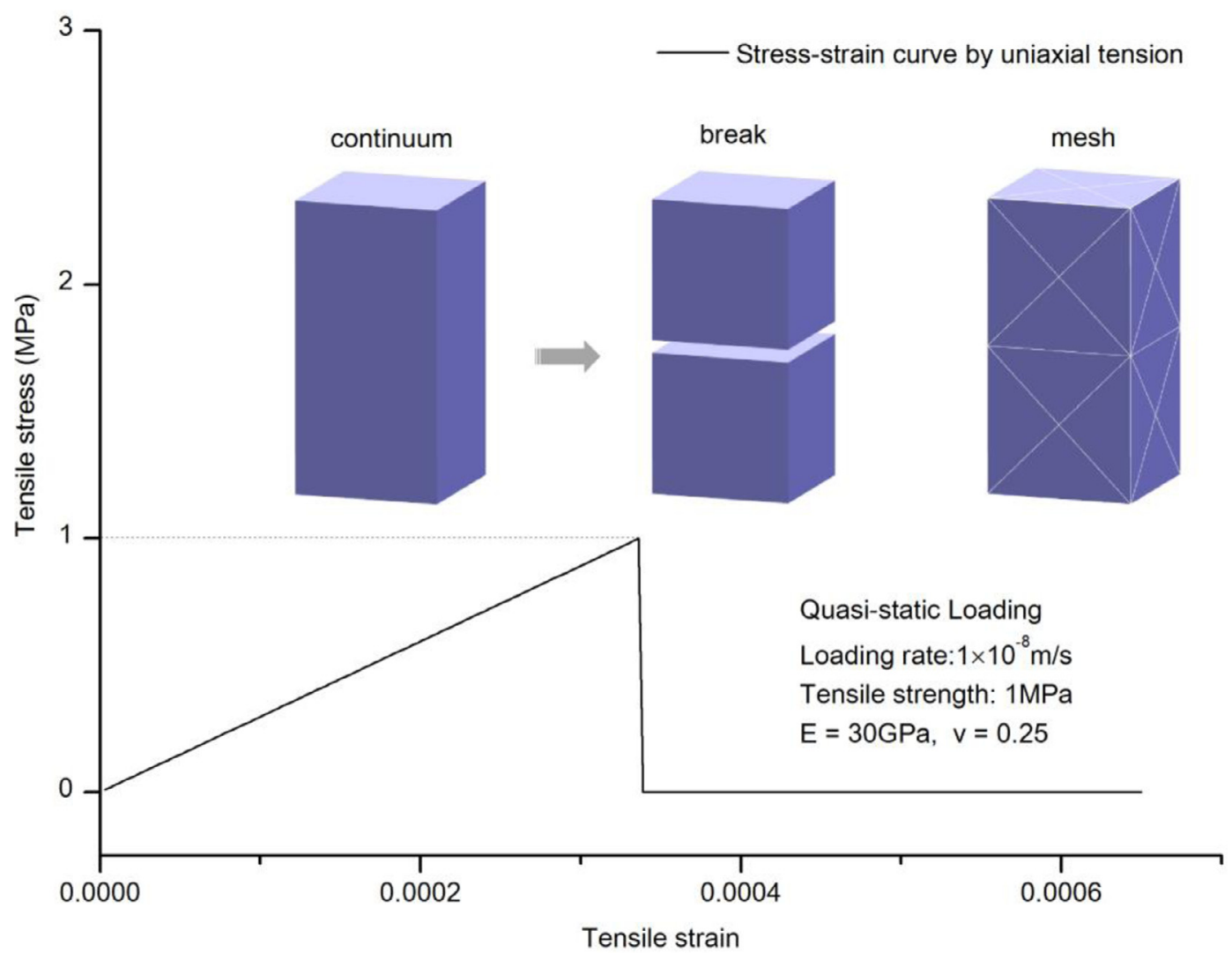

Fig. 5. Tensile breakage of a block. The model consists of 48 tetrahedron elements. The longitudinal degree of freedom for the bottom of the model is fixed, while a constant longitudinal displacement loading is applied on the upper surface. Failure of the model takes place exactly at the point of the tensile strength.

The characteristic time $T_{e}$ is defined as

$$
T_{e}=2 \pi \sqrt{m / K}
$$

where Kis the stiffness of the subdomain.

Define $t=T_{e} t^{\prime}$, Eq. (22) can be written as

$$
\ddot{u}_{i}+\frac{2 \pi c}{\sqrt{m K}} \dot{u}_{i}+\frac{4 \pi^{2}}{K} F_{i}^{\varepsilon}=\frac{4 \pi^{2}}{K} F
$$

The dimensionless damping coefficient $\zeta$ can be written as

$$
\zeta=\frac{2 \pi c}{\sqrt{m K}}
$$

Thus, the damping coefficient is

$$
c=\frac{1}{2 \pi} \zeta \sqrt{m K}
$$

The dimensionless damping coefficient determines the attenuation process of the vibration. Usually, it can be obtained through standard tests. Otherwise, it can also be determined by inversion algorithm with calculation if the vibration curve of a structure is given in a test.

\subsection{The time step}

In this method, time step is not only limited to the frequency of the external forces, but also depends on the natural vibration period of the structure. The scale of the subdomain is a characteristic scale. In order to ensure the reliability and convergence of the calculation, the distance of the wave propagation within a time step should be less than the characteristic scale of the subdomain. Otherwise, it may result in computational divergence. 


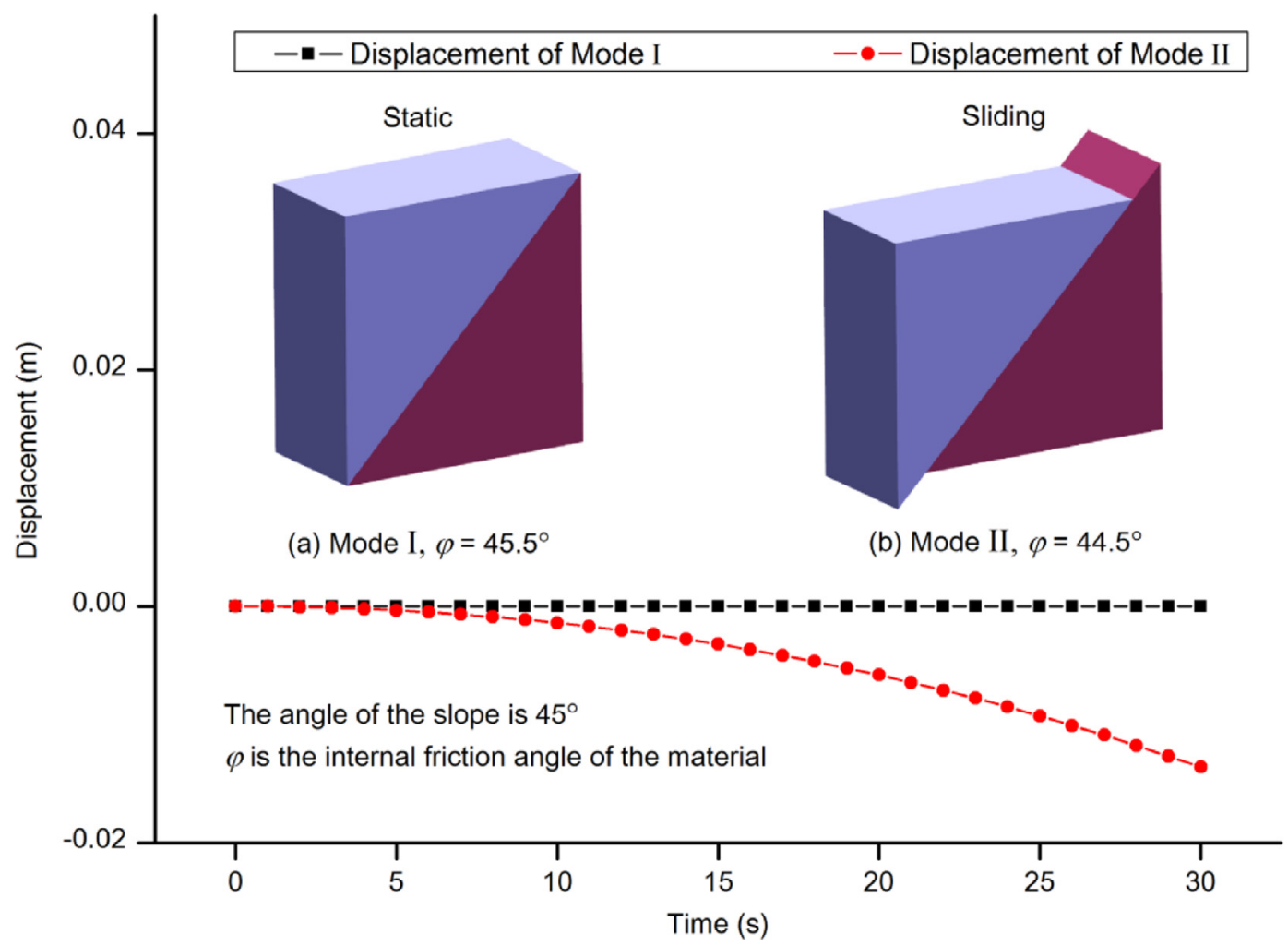

Fig. 6. Contact and friction between two blocks. The slope of the model is $45^{\circ}$. When the internal friction angle of the material $\varphi=45.5^{\circ}$, which is a little larger than the slope, the block is static. However, when $\varphi=44.5^{\circ}$, the block is sliding.

The minimum inherent period of the nodes in the system is defined as the characteristic time, which can be written as

$$
T_{e}=2 \pi \sqrt{\frac{m}{K}}
$$

where $m$ is the mass of the node, $K$ is the stiffness of the related subdomain.

In the meantime, when the frequency is high, such as the problem of blasting, the period of the external force $T_{f}$ should be considered. So, the time step should satisfy the following condition.

$$
\Delta t \leq \min \left(T_{e}, T_{f}\right)
$$

\subsection{Fracture criterion}

Nature of the failure process for brittle material is the growth and coalescence of internal micro cracks and micro defects until macro structural failure takes place. Theories of plasticity use nonlinear constitutive models and relevant failure criteria to describe this physical process. Internal fractures and crack growth within the material cannot be described clearly with plastic model. Fracture mechanics based on stress intensity factor focuses on the mechanical behavior around the tip of a crack, which is a great tool to investigate the behavior of crack growth. But it's quite hard to use it to describe the failure process of a heterogeneous material or a practical structure, since the fracture state within the material is complex and fracture mechanics usually concentrates on single-crack property. Moreover, the result of the fracture mechanical model greatly depends on the element size.

The complex macro mechanical behavior of the brittle material is originated from the internal fractures, and the macroscopic nonlinear stress-strain relationship can be naturally obtained though elastic deformation of the intact parts and the mesoscopic interactions of discontinuities within the material body [24]. Thus, simple constitutive model and fracture criteria are used in PSM based on the conception "simple constitutive model, complex geometric construction".

In this model, there are two basic failure modes, tensile failure and shear failure. Fracture of the material under other stress or strain conditions, such as bending and compression, can be seem as the combination of the two basic failure modes. Thus, the maximum tensile strength and the shear strength based on the maximum tensile stress criterion and a simplified Mohr-Coulomb criterion $[7,8,26,30,38]$ are used in this paper. When evaluating the fracture criteria, the stress at the central node is defined as the average stress of the subdomain. When the first principal stress of the subdomain exceeds the tensile strength, tensile failure takes place. Shear failure comes when the maximum shear stress exceeds the 

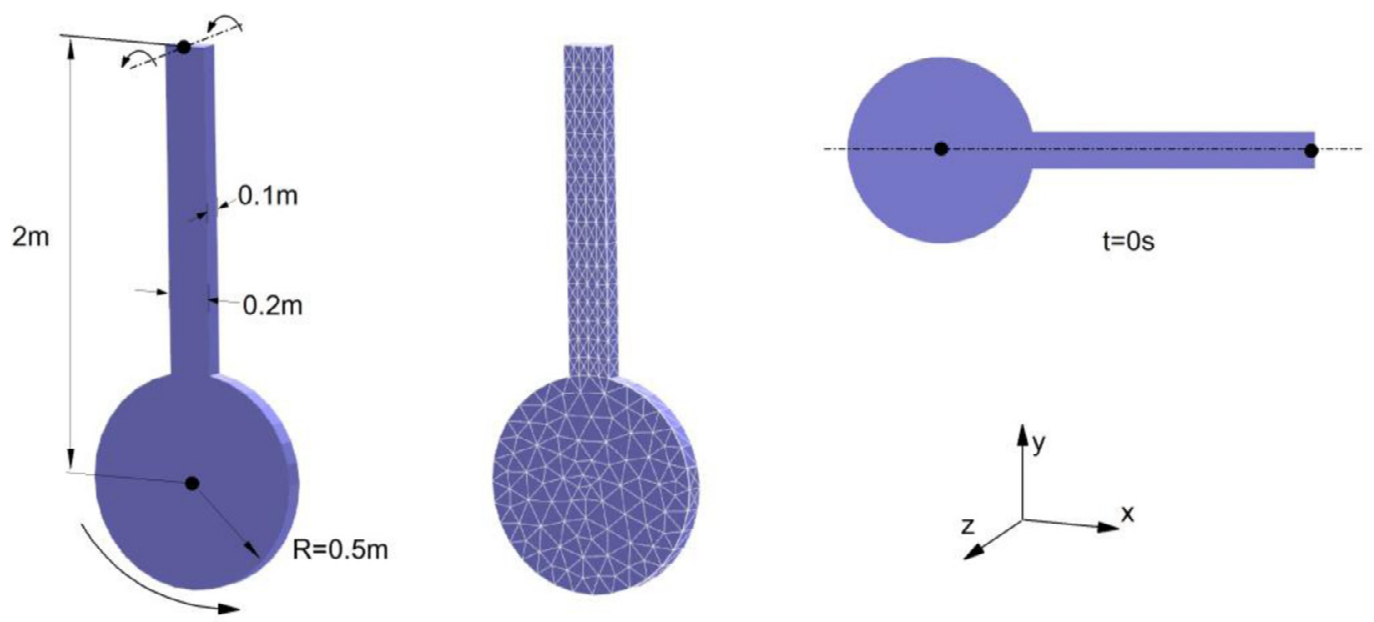

(a) Model of the compound pendulum

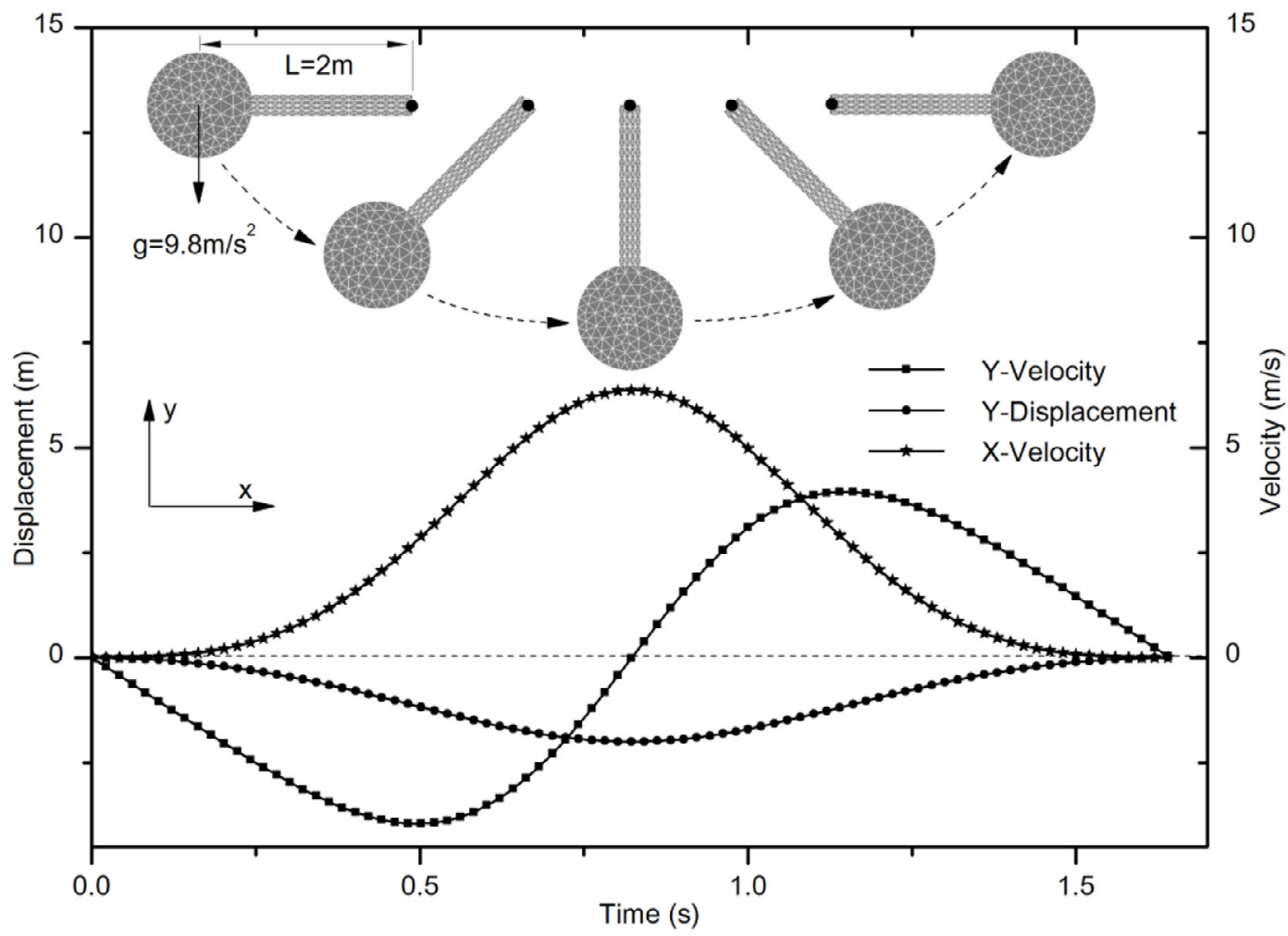

(b) Result of the simulation

Fig. 7. Motion of the compound pendulum. The length of the compound pendulum is $2 \mathrm{~m}$ from the end the beam to the center of the disk. The pendulum will swing freely around the fixed point under gravity without damping. The velocity and displacement at the center of the disk are shown, which fit well with the theoretical resolutions.

shear strength according to Mohr-Coulomb criterion [22]. So cracks could occur in the computational domain when meeting either of the following conditions,

$$
\begin{aligned}
& \sigma_{1} \geq \bar{\sigma}_{\mathrm{T}} \\
& \tau_{\max }=\frac{\sigma_{1}-\sigma_{3}}{2} \geq \sigma_{\mathrm{n}} \tan \varphi+C
\end{aligned}
$$




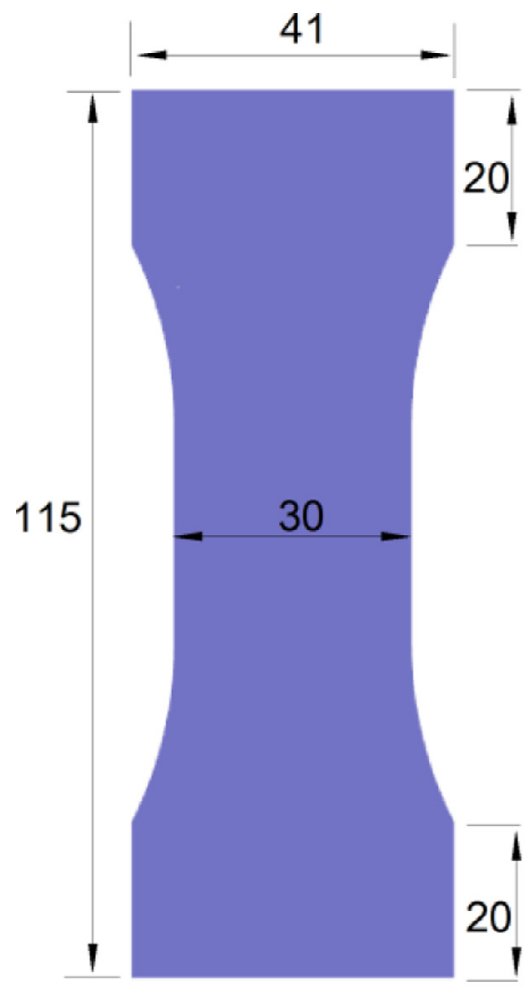

(a) Size of the model

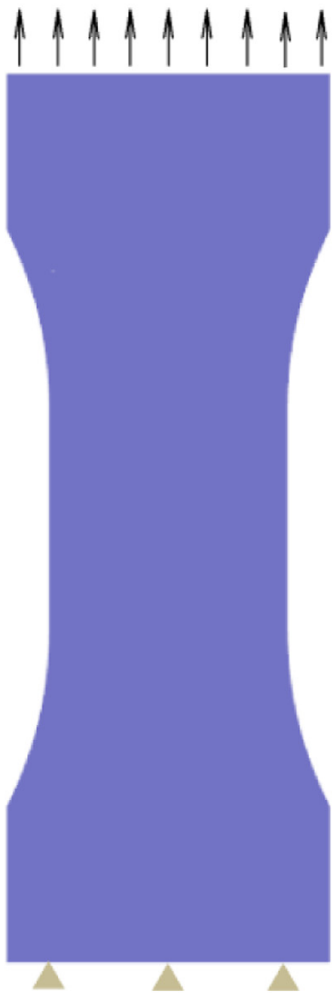

(b) Boundary conditions

Fig. 8. Size(mm) and boundary conditions, specimen used by Mohamed and Farzaneh [25].

Where $\sigma_{1}$ is the first principal stress of a subdomain, $\bar{\sigma}_{\mathrm{T}}$ is the maximum tensile strength, $\tau_{\max }$ is the maximum shear stress and $\sigma_{\mathrm{n}}$ is the normal stress on the plane of the maximum shear stress, $\varphi$ is the internal friction angle, $C$ is the cohesion value of the material.

Moreover, any other criterion could be used to evaluate the fracture considering the applicability to distinct materials and problems. The particle as well as the subdomain will be divided into two new ones, when the stress on the interface of the elements within the original subdomain exceeds the strength, as shown in Fig. 3. The new born crack will be a new boundary within the computational domain. During the failure process, the position and type of the contact will be detected and the contact force will be calculated to describe the interaction of the cracks within the material and the motion of the fragments with discrete element method.

\subsection{Computational procedure}

With the theory and technology introduced above, PSM could resolve plenty of problems relating to the dynamic, fracture and motion. The code for PSM has been written and implemented in $\mathrm{C}++$ language. The iteration process is based on the dynamic relaxation. Movement of the particle system is based on the dynamic equation derived from the Lagrange equation, as shown in Eq. (21). The forces of the particles consist of internal force produced by the subdomain with associate elements, body force and external force. The internal force of a subdomain is calculated with the finite element method. Given fracture criterion, a subdomain can break along the interface of the associate elements, when exceeding the strength. Then, the original subdomain and particles will be divided into two new ones by the crack surface. The new born crack is a new boundary within the computational domain, and interactions at the crack are described with contact and friction. During the fracture process, the original continuous domain may break into some individual blocks. The minimum size of a block is an element. As long as the stiffness matrix of each element keeps updating with the time and position, the movement and rotation of blocks can be accurately expressed with the equation of motion (21).

The computational procedure of PSM is:

i) Mesh the computational model and determine the initial subdomain for each particle, determine the boundary condition and initial condition for the model;

ii) Generate the element stiffness matrix for each element and assign mass to each particle according to the associate subdomain; 


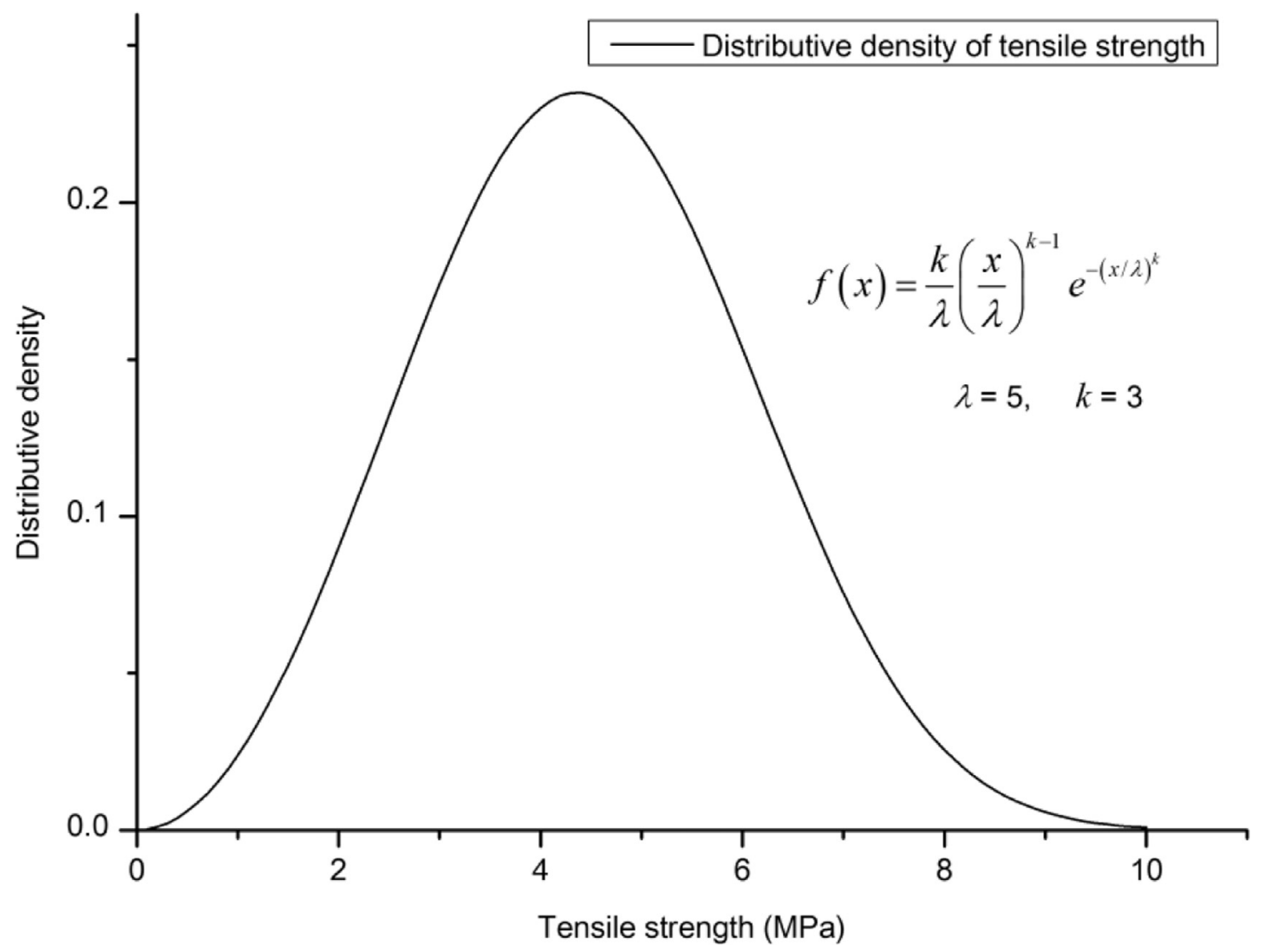

Fig. 9. Distribution of the tensile strength with Weibull distribution.

iii) Calculate the internal force of the subdomain with the finite element method using Eqs. (19) and (20);

iv) Detect the contact information at the boundaries, and determine the contact force. The contact force will be regarded as external force added to the associate particles.

v) Loop over all the particles and subdomains, calculate the acceleration of each particle with equation of motion (21), and then calculate the velocity and displacement of the nodes within a time-step;

vi) Evaluate the stress state in the subdomains to judge if the fracture criterion has been met, and that may produce new particles, subdomains and boundaries;

vii) Renew the particle and subdomain system, update the coordinate of the particles to the current position, and then renew the element stiffness matrixes as well;

viii) End the current time-step and start the calculation for the next time-step;

ix) Loop from step (iii) to (viii), until meeting the terminal condition.

It's worth mentioning that the method is 3D in its origin. The theories and techniques are suitable for both 2D and 3D. 2D simulation can be realized with a single layer of elements in thickness and displacement constrain in thickness direction. And FEM is not the only way to calculate the internal force of a subdomain. Any other continuous model can be used. The core concept of PSM is a dynamic algorithm with dynamic relaxation to solve a particle system with associate subdomains, considering fracture, contact and motion. The continuous computational domain can be easily changed to discrete blocks composed of new generated particles and boundaries along the interfaces of the elements. Once blocks are generated, contacts at the cracks or boundaries can be described with DEM. Since the mesh keeps updating during the computational process with fracture and movement of the system, mesh distortion can be completely avoided. PSM has combined the advantages of particle-in-cell method, continuous element method (i.e. FEM and FVM) and discrete element method. Failure process from continuous deformation, crack nucleation, fracture evolution and motion of blocks can be expressed and described with a unified form.

\subsection{Verifications}

Simple numerical examples are demonstrated to verify this method. Fig. 4 shows the wave propagation process in a beam calculated with PSM. Size of the beam is $5 \mathrm{~m} \times 5 \mathrm{~m} \times 60 \mathrm{~m}$. Velocity of a half sinewave is applied to the left end 


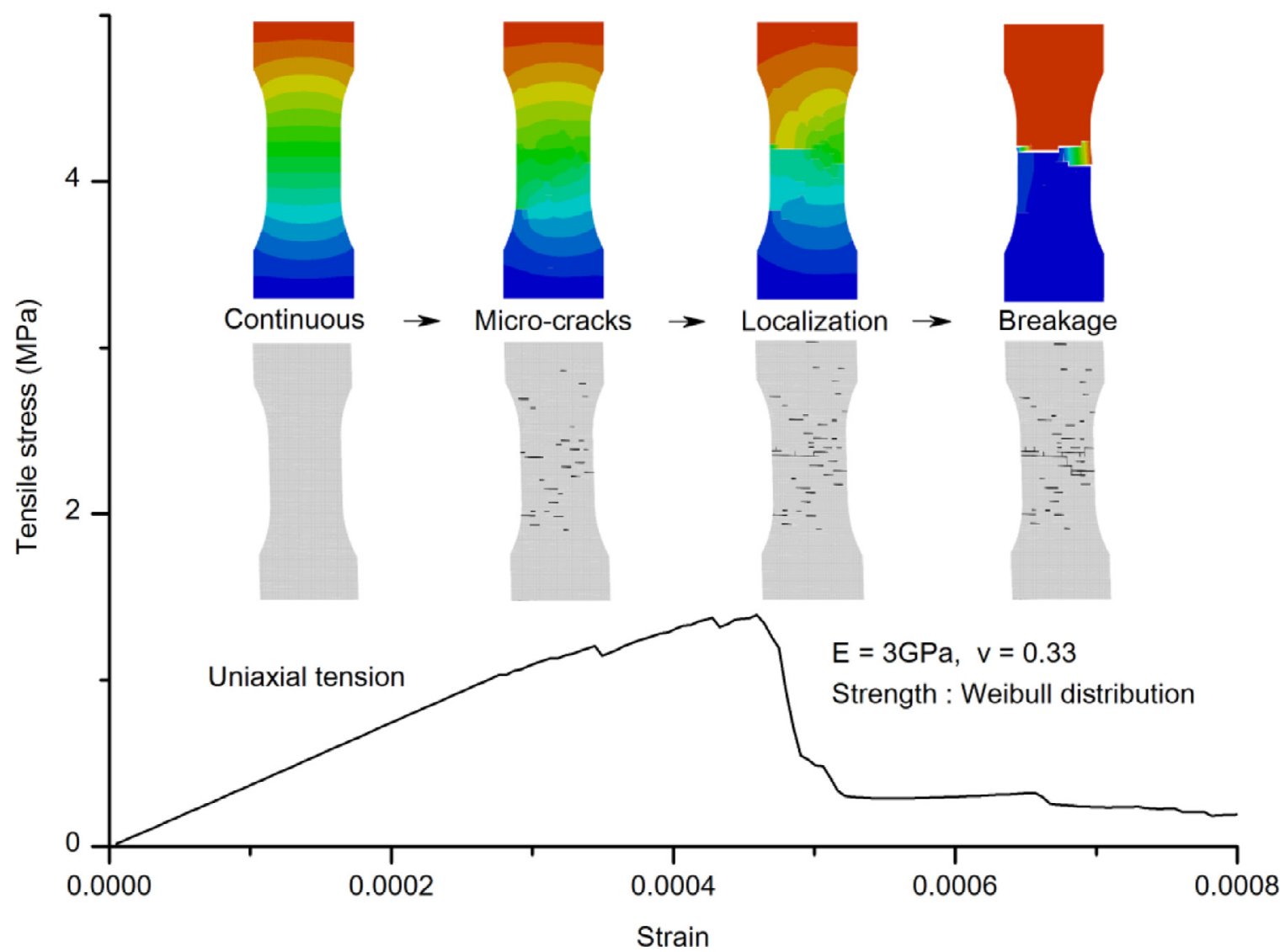

Fig. 10. Stress-strain curve under uniaxial tension and the fracture process of the specimen. Failure of the specimen consists of linear elastic stage with continuous deformation, nonlinear stage with homogeneous micro-cracks, strain softening stage with cracks localization and the breakage stage with one or several principal cracks.

of the free beam with the amplitude of $10 \mathrm{~m} / \mathrm{s}$ and the period of $0.1 \mathrm{~s}$. During the computational process, wave in the beam will propagate with a specific velocity depending upon the material property. Theoretical expression for the velocity of longitudinal wave in a material is

$$
V_{c}=\sqrt{\frac{(1-v) E}{(1+v)(1-2 v) \rho}}
$$

Where $V_{c}$ represents the velocity of the longitudinal wave, $E$ is the Young's modulus, $\rho$ is the density, $v$ is the Poisson's ratio. Wave velocity calculated by PSM is $3.79 \times 10^{3} \mathrm{~m} / \mathrm{s}$, which agrees with the theoretical value precisely. It demonstrates that PSM is effective to solve problems with dynamic and wave propagation.

Fig. 5 shows the tensile failure of a simple model. The model is composed of 48 tetrahedron elements. With uniaxial tension, the interfaces in the middle of the model break at the point of tensile strength 1MPa precisely. Fig. 6 shows the contact and friction between two blocks. The slope is $45^{\circ}$. When the internal friction angle is larger than the slope, as model I with $\varphi=45.5^{\circ}$, the block keeps still. When the internal friction angle is less than the slope, as model II with $\varphi=44.5^{\circ}$, the block will slide. Numerical results show remarkable consistency with the theoretical condition. Fig. 7 shows the motion of the compound pendulum. The length of the compound pendulum is $2 \mathrm{~m}$ from the end of the beam to the center of the disk. The size and mesh are shown in Fig. 7 (a). The Young's modulus $E$ is $210 \mathrm{MPa}$. The Poisson's ratio $v$ is 0.25 . The density is $7800 \mathrm{~kg} / \mathrm{m}^{3}$. The pendulum will swing freely around the fixed point under gravity without damping on the XY plane. The theoretical value of the velocity and displacement at the center of the weight can be calculated through the energy conservation between gravitational potential energy and kinetic energy as

$$
m_{1} g h_{1}+m_{2} g h_{2}=\frac{1}{2} m_{1} v_{1}^{2}+\frac{1}{2} m_{2} v_{2}^{2}
$$

Where $m_{1}$ is the mass of the rod, $m_{2}$ is the mass of the weight, $h_{1}$ and $h_{2}$ are the vertical falling distance for the center of the rod and the weight respectively, $v_{1}$ and $v_{2}$ are the velocity for the center of the rod and the weight respectively. When the weight moves to the button, the displacement in $\mathrm{Y}$ direction for the center of the weight is $2 \mathrm{~m}$, the velocity in $\mathrm{Y}$ direction 


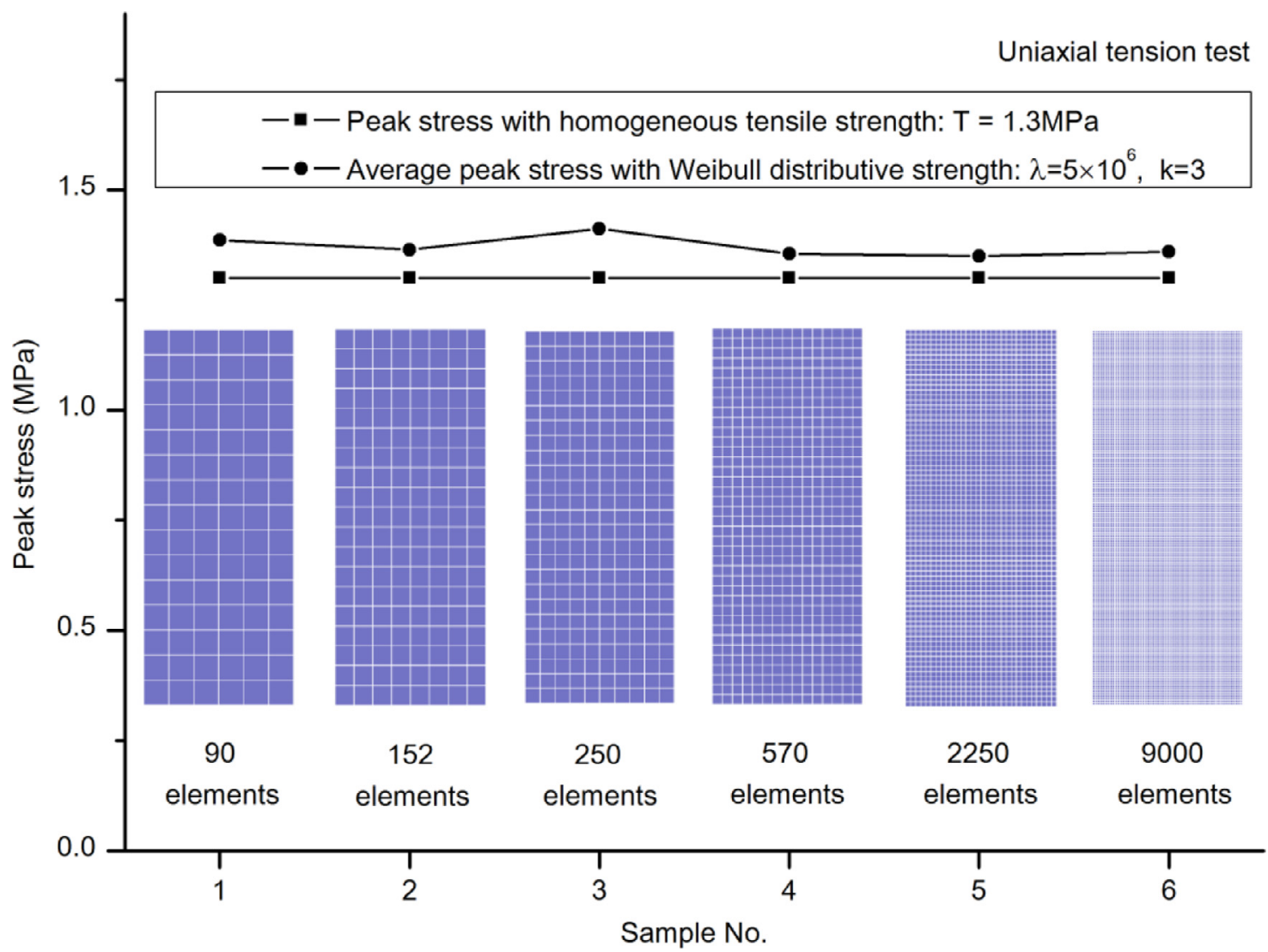

Fig. 11. The effect of element size on tensile strength. Under quasi-static loading, the peak stress is almost not effected by the size of element either with homogeneous strength or distributive strength.

is $0 \mathrm{~m}$ and the velocity in $\mathrm{X}$ direction is about $6.37 \mathrm{~m} / \mathrm{s}$. The computational results fit well with the theoretical resolutions, which validate that PSM is applicable to the problems with large displacement, motion and rotation.

\section{Numerical modeling for brittle failure of ice}

\subsection{Ice parameters}

Lots of tests for ice have been performed in the lab to investigate the material property of ice. However, the tests show a wide range of scatter for the mechanical parameters. The tensile strength in the published investigations is ranging from 0.7 to $3.1 \mathrm{MPa}$. And the average tensile strength of ice is about $1.43 \mathrm{MPa}$ at the temperature from $-10{ }^{\circ} \mathrm{C}$ to $-20^{\circ} \mathrm{C}$ [4]. Typical value of the compressive strength ranges from $0.5 \mathrm{MPa}$ to over $5 \mathrm{MPa}$, and is a strong function of loading strain rate and less dependent on the temperature until close to the melting point [41]. The typical measured values of elastic modulus are from $1 \mathrm{GPa}$ to $10 \mathrm{GPa}$ ([25]). The Poisson' ratio ranges from 0.33 to 0.42 [28]. The internal friction coefficient (ice to ice) is about 0.05 to 0.2 according to the report of Frederking and Barker [13]. The variation of strength mainly depends on temperature, micro-structure, grain size, specimen size and loading rate, etc. Complex mechanical behaviors of ice are determined by the complex properties. Numerical modeling for the brittle failure of ice in the laboratory scale is scarce, primarily because of the challenge of the complex characteristics of ice and the demand for more advanced numerical methods. In this study, the brittle behaviors of the ice specimen are modeled with PSM introduced above. The variation of the parameters, complex internal structures and components of ice are described by the distribution of strength within the material.

\subsection{Tensile failure}

Tensile failure is a common phenomenon in natural ice and the tensile strength is a fundamental parameter for ice. However, just very few experiments have been performed to measure the tensile strength because of the complex brittle property of ice and high demands for the test set up. Numerical simulations for the tensile test of ice are much scarcer, because it's quite hard to use the current numerical methods to describe the brittle failure process of ice precisely, since the process includes continuous deformation, distributive micro-cracks initiation, cracks localization and rupture. 


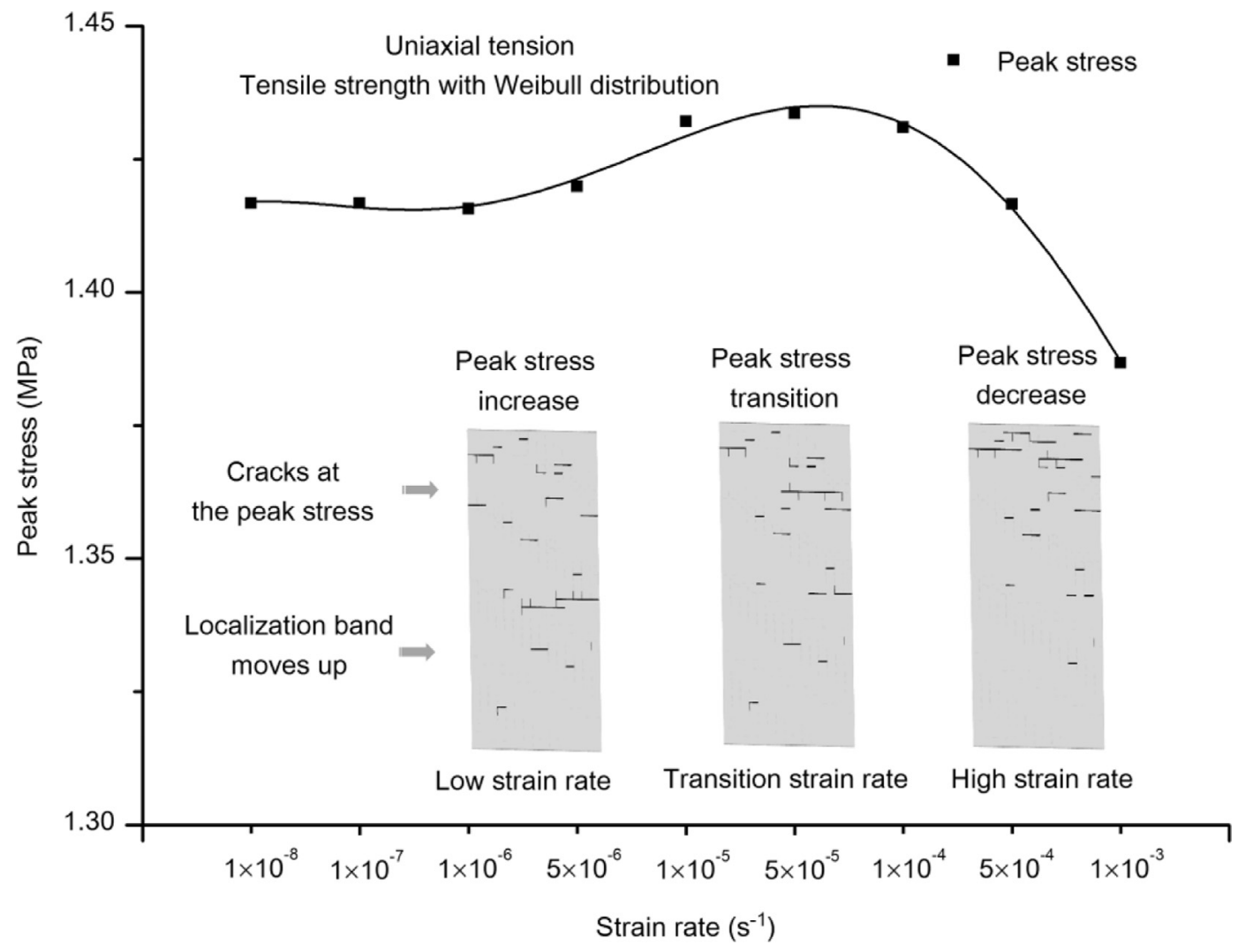

Fig. 12. Peak stress and crack position under different strain rates. At very low strain rate, i.e. below $10^{-6} \mathrm{~s}^{-1}$, peak stress of uniaxial tension keeps constant, and the localization band will be initialized in the weakest part of the specimen. When the strain rate is between about $10^{-6}-10^{-4} \mathrm{~s}^{-1}$, peak stress will increase with the decrease of the strain rate, and the localization band is more likely to appear in the upper half of the specimen. However, if the strain rate continues to increase, i.e. above $10^{-4} \mathrm{~s}^{-1}$, the peak stress will decrease with the increase of the strain rate, and the fracture is concentrated at the top close to the loading surface.

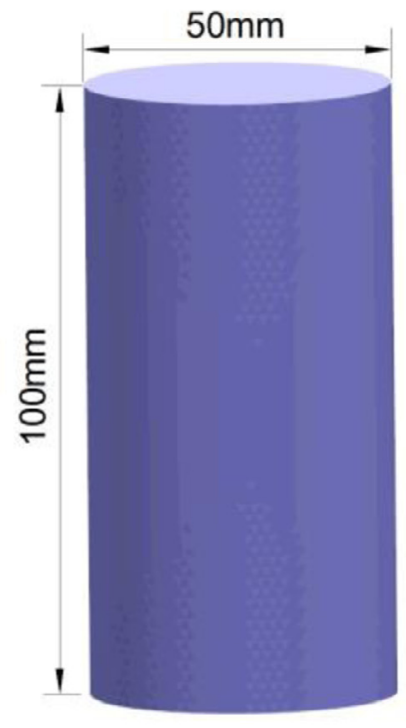

model and size

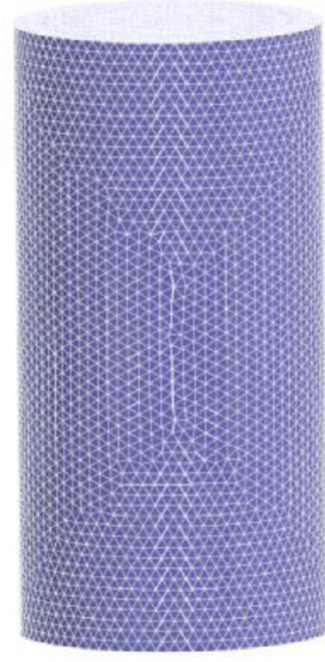

224,847 elements

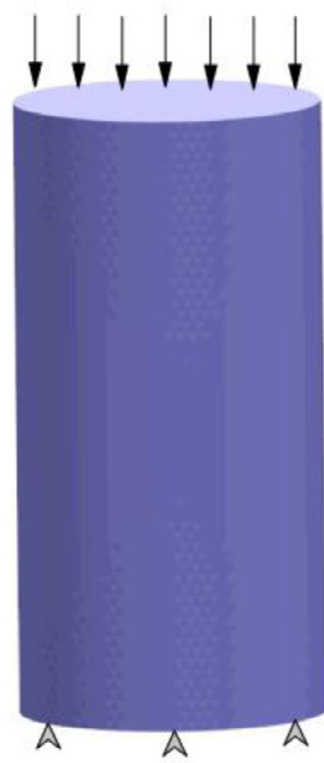

boundary conditions

Fig. 13. Numerical model of the compressive test. 

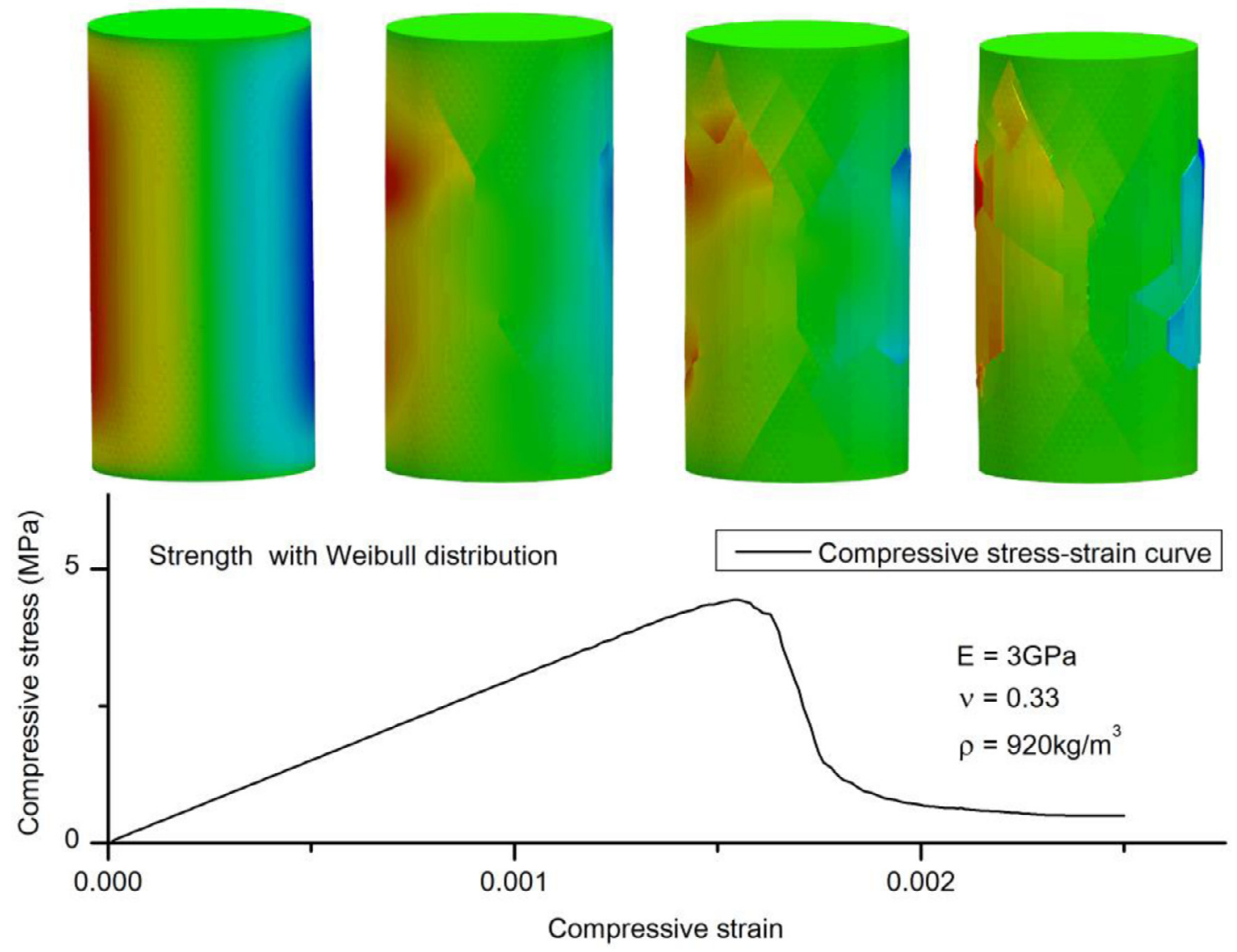

Fig. 14. Failure process of the specimen and the stress-strain curve during compression.

Table 1

Key parameters used for uniaxial tension. The tensile strength complies with Weibull distribution law with scale parameter $\lambda$ and shape parameter $k$.

\begin{tabular}{lllllll}
\hline$E(\mathrm{GPa})$ & $v$ & $\rho\left(\mathrm{kg} / \mathrm{m}^{3}\right)$ & $\frac{T}{2}$ & $L(\mathrm{~mm})$ & $V\left(\mathrm{~s}^{-1}\right)$ \\
\cline { 3 - 6 } & & & $\lambda$ & $k$ & & \\
\hline 3 & 0.33 & 920 & $5 \times 10^{6}$ & 3 & 0.7 & $1 \times 10^{-7}$ \\
\hline
\end{tabular}

PSM provides a new way to resolve these problems. Numerical model of the tensile test is established according to the work of Mohamed and Farzaneh [25]. The specimen size and the boundary condition are shown in Fig. 8. and parameters used for this model is listed in Table 1.

$E$ is the Young' modulus, $v$ is the Poisson' ratio, $\rho$ is the density of ice, $T$ is the tensile strength, $L$ is the mesh size, $V$ is the strain rate. The tensile strength is distributed in the model to describe the heterogeneity of ice material. Each node and subdomain will get a random strength according to Weibull distribution [46] with parameter $\lambda$ and $k$. The distributive density function can be written as

$$
f(x)=\frac{k}{\lambda}\left(\frac{x}{\lambda}\right)^{k-1} e^{-(x / \lambda)^{k}}
$$

With $\lambda=5, k=3$, the distribution of tensile strength within this model can be obtained as Fig. 9.

The stress-strain curve of the specimen under uniaxial tension at the strain ratio $1 \times 10^{-6} \mathrm{~s}^{-1}$ is shown in Fig. 10 . Failure of the specimen under uniaxial tension has gone through 4 stages, which include linear elastic stage, nonlinear stage, strain softening [33] stage and breakage stage. Internal facture state of the specimen has got specific characteristics during each stage as shown in Fig. 12. In the linear elastic stage, the material is intact with elastic deformation. In the nonlinear stage, micro-cracks within the material are homogeneously generated due to the distribution of strength. Taken in this sense, nature of the macro nonlinear behavior of brittle material is caused by internal mesoscopic or microscopic damage and fracture. During the strain softening stage, coalescence of the micro-cracks within the material starts to form a localization 

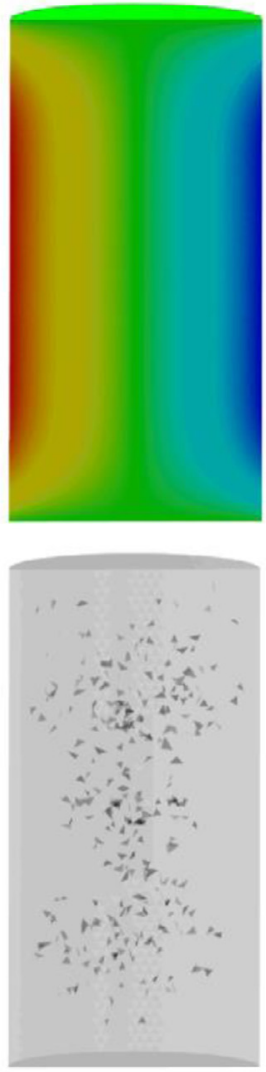

Homogeneous micro-cracks
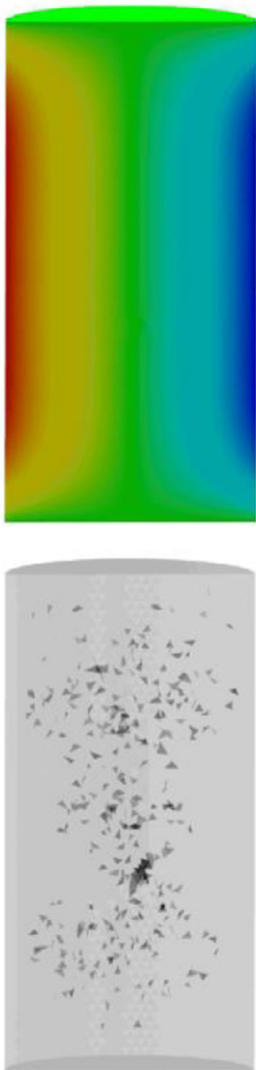

Principal crack

nucleation

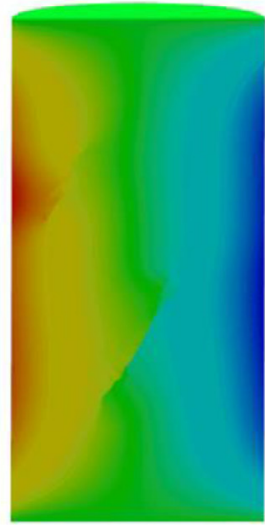

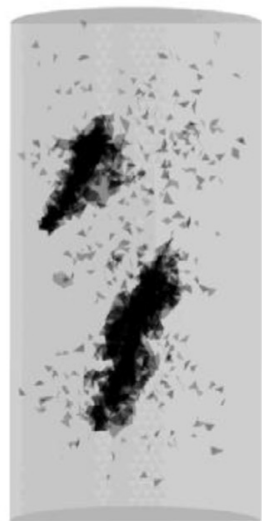

Localization bands generation
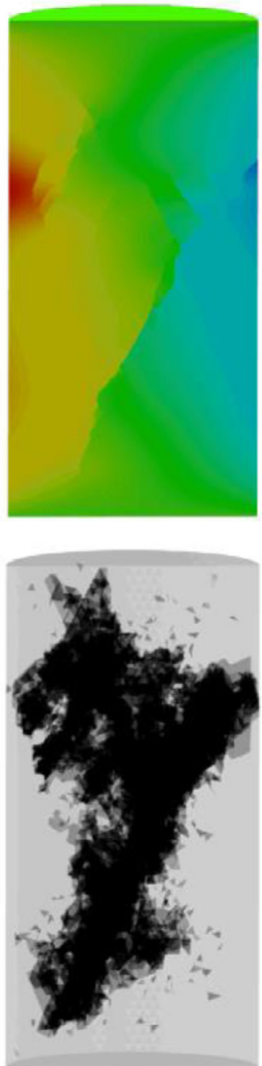

\section{Fracture further evolution}

Fig. 15. Fracture evolution within the cylindrical specimen. The specimen has been cut into halves, the cracks on the middle section can be observed through the cutaway view and the internal micro cracks evolution can be seen through the perspectives.

band. Thus, factures will focus on this localization band due to stress concentration. In the last stage, the specimen is totally failed with one or several principal cracks.

In order to further validate and investigate the element size effect and loading rate effect, a simplified model is used with $30 \mathrm{~mm}$ in width and $75 \mathrm{~mm}$ in length, since in numerical modeling, the uniaxial boundary condition can be easily added to the model without special handling for the ends of the specimen. Under quasi-static loading condition, peak stress is almost not affected by the element size as shown in Fig. 11. However, since the strength of the material complies with a certain distributive law, increase of the element number will reduce the scatter of the results and produce more reasonable outcomes. But on the other hand, the size of the elements could represent the size of grain, because the characteristic length of cracks is the scale of the elements in PSM. Thus, it is more reasonable to choose the element size according to the grain of ice.

Loading rate will influence the tensile strength of the specimen as shown in Fig. 12. The tendency is in accord with the result of Mohamed and Farzaneh [25]. Under quasi-static loading, when the strain rate is quite low, i.e. under $10^{-6} \mathrm{~s}^{-1}$, the peak stress keeps constant. Under this condition, the specimen can be regarded as maintaining in the static equilibrium state at any moment during the loading process, crack initiation will occur at the weakest point within the material. There is no strain rate dependency observed in this stage. With the increase of the strain rate, the peak stress increases. This happens because the loading condition should no longer be regarded as quasi-static any more. Deformation close to the loading-end is larger than that in the far-end. So the average stress in the upper half is a bit larger than that in the lower half of the specimen. Under this circumstance, crack initiation will not start at the weakest part of the specimen, but at some other position in the upper half where the strength is stronger due to stress inhomogeneous. That's why the localization band tends to appear at the upper half of the specimen. And this may result in the increase of the macro tensile strength in the test. If the strain rate continues to increase until exceeding a certain value, i.e. above $10^{-4} \mathrm{~s}^{-1}$ in this case, the peak stress will decrease with the increase of the strain rate. This is caused by the high speed loading condition. Stress inhomogeneous is more obvious. High strain rate of the loading-end would lead to the deformation and energy accumulation close to the 


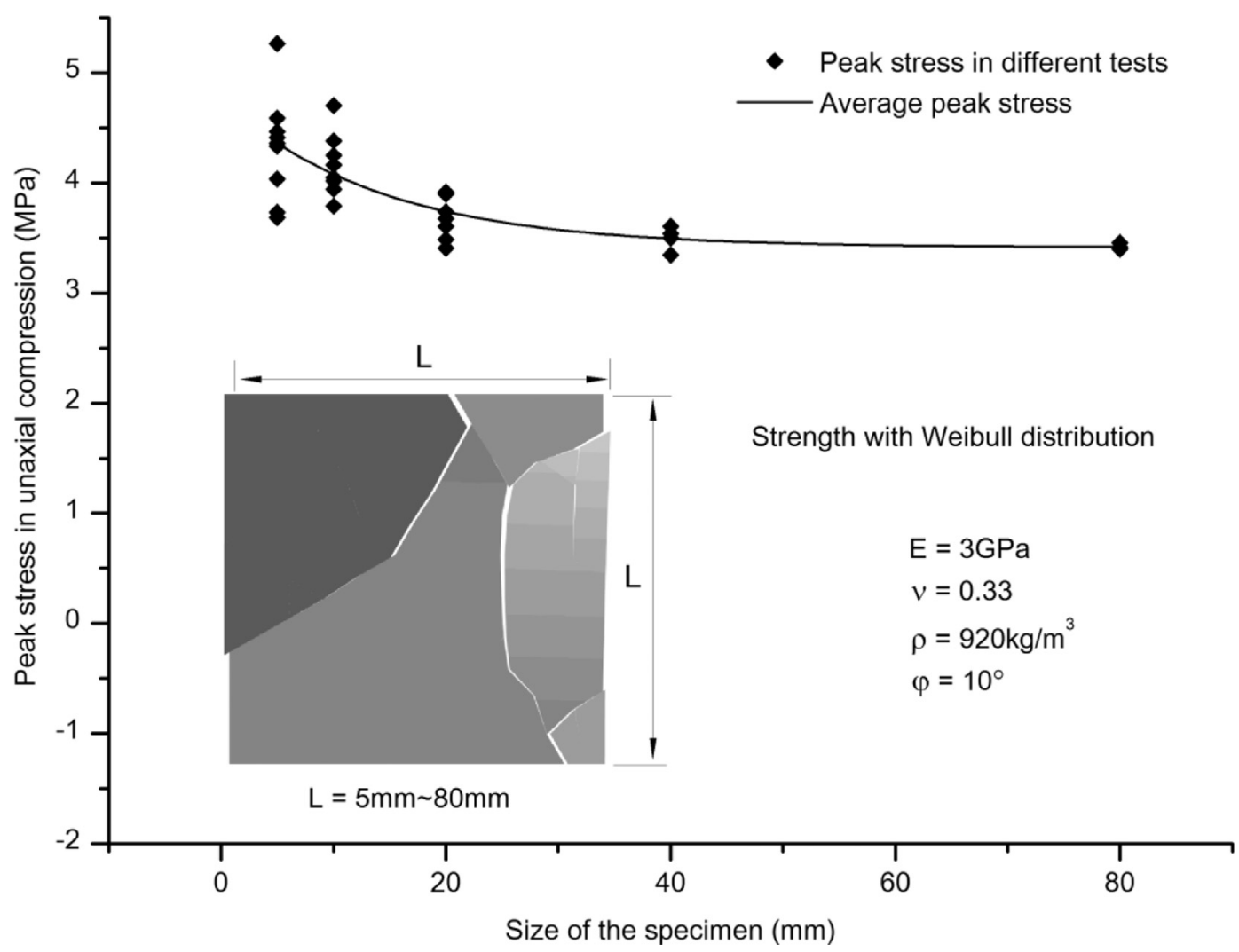

Fig. 16. Peak stress vs. specimen size. Specimens with length of $5 \mathrm{~mm}, 10 \mathrm{~mm}, 20 \mathrm{~mm}, 40 \mathrm{~mm}$ and $80 \mathrm{~mm}$ are tested with PSM. The average peak stress will increase when the size decreases. If the size is large enough, i.e. above $20 \mathrm{~mm}$ in this case, the average peak stress is independent of specimen size.

Table 2

Weibull parameters for the distributive strength used in the 3D compressive test.

\begin{tabular}{lllllll}
\hline$C$ & & $T$ & & \multicolumn{2}{l}{} \\
\cline { 3 - 4 }$\lambda_{C}$ & $k_{C}$ & $\lambda_{T}$ & $k_{T}$ & & $\lambda_{\varphi}$ & $k_{\varphi}$ \\
\hline $4 \times 10^{6}$ & 15 & $4 \times 10^{6}$ & 15 & & 10 & 15 \\
\hline
\end{tabular}

loading surface. Rapid increase of the stress at the top will result in a large number of cracks generated at the very beginning close to the loading surface. Energy dissipation of the fractures will reduce the energy and stress wave propagation to the far-end. Failure of the specimen is manifested as local failure close to the top. So the global strength of the specimen would show the phenomenon of decrease when local fracture takes place at the very beginning under this condition.

However, the variation range of tensile strength cause by strain rate is not profound comparing to compression, which will be discussed in the following section.

\subsection{Compressive failure}

Brittle compressive failure is more complicated than tensile failure, since the failure process may include tensile fracture, shear fracture and friction. Meanwhile, it is another very important failure mode in nature. Ice islands, icebergs and thick ice sheets against each other or against off-shore structures will result in brittle compressive failure [38]. Lots of tests have been performed to investigate the compressive property of ice. Numerical model used here is a cylinder with diameter 50 mm and length $100 \mathrm{~mm}$, as shown in Fig. 13. The mesh size is $2 \mathrm{~mm}$, which is chosen to be the same scale as the grain size. The bottom of the specimen is fixed and the horizontal movement of the top is constrained. Constant longitudinal displacement loading will be applied on the top surface.

Fracture criterion used in this model is introduced in Section 2.3. Young' modulus of the specimen is 3 GPa. Poisson's ratio is 0.33 . The density of ice is $920 \mathrm{~kg} / \mathrm{m}^{3}$. In order to describe the property of heterogeneity of natural ice, cohesion, tensile strength and internal friction angle comply with Weibull distribution. The parameters are shown in Table 2.

Stress-strain curve under quasi-static loading is show in Fig. 14. Under quasi-static loading, the progressive failure process is relatively slow. Strain softening behavior is obvious. The fracture evolution process within the material is shown in 


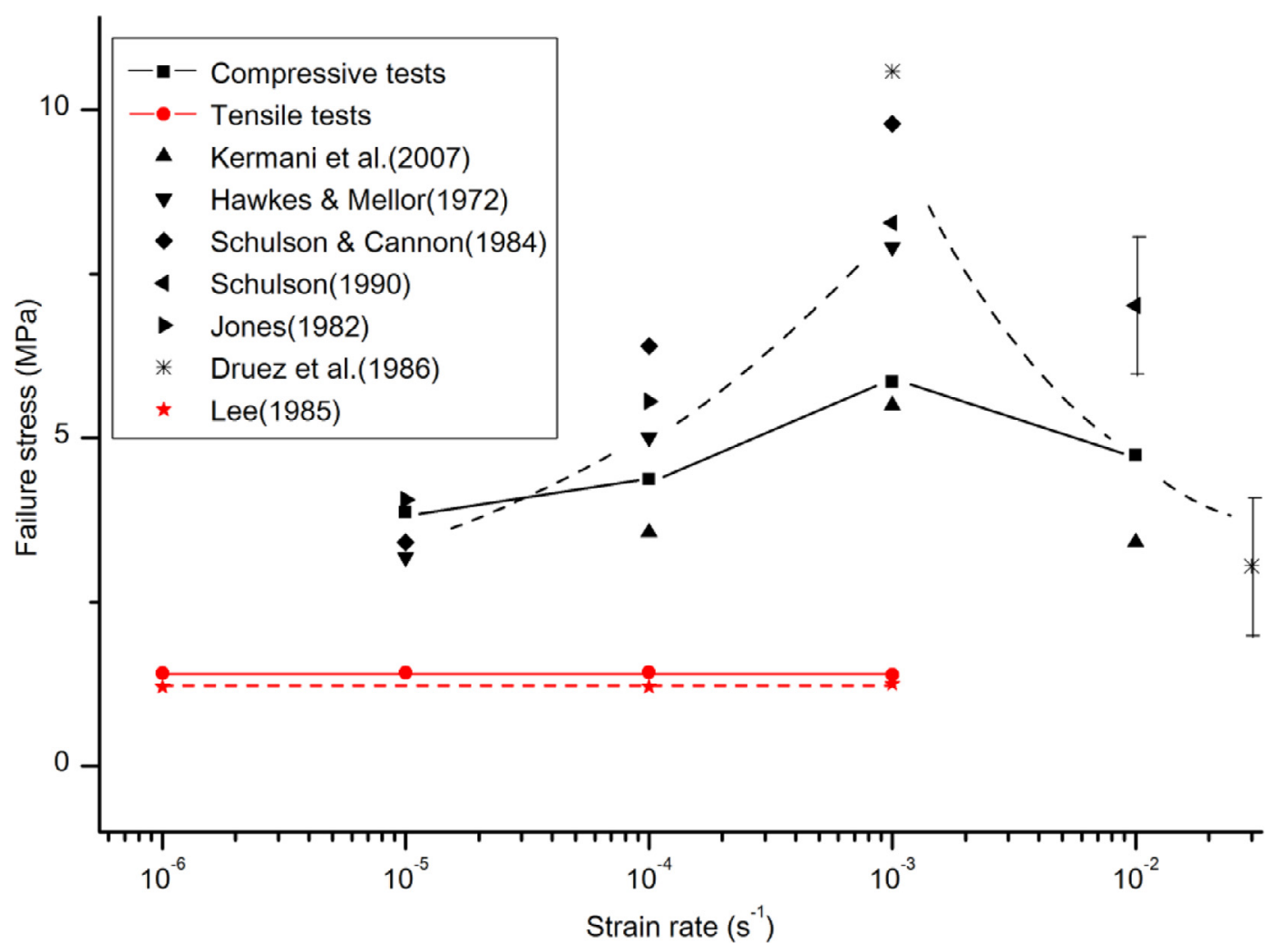

Fig. 17. Failure stress vs. strain rate. Compressive strength is remarkably influenced by strain rate. When the strain rate is below about $10^{-3} \mathrm{~s}^{-1}$, compressive strength will increase rapidly with the increase of the strain rate. However, above about $10^{-3} \mathrm{~s}^{-1}$, the strength will decrease with the increased of the strain rate. Numerical and experimental results of tension are also shown for comparison.

Fig. 15. The specimen has been cut into halves in order to observe the internal fracture state during the compressive failure process. From the cutaway view of the axial section, one could see that the principal cracks are about $30^{\circ}$ deviating from the vertical axis. These cracks are mainly caused by shear fault accompany with local splitting (See Fig. 14). Development of internal fractures can be easily seen from the perspectives in Fig. 15. At the very beginning, plenty of micro-cracks will be homogeneously generated within the material until principal crack nucleation occurs at the weakest parts. Since then, localization bands will gradually take shape and fractures will focus on these localization bands until the global failure happens. The four stages of compressive failure are the same as tensile failure. But there are two critical differences during the failure process that may remarkably affect the mechanical property and distinguish compressive failure and tensile failure. One is the shear fault, and the other is friction.

Size effect is studied through a quadrate plane strain model with random mesh and strength. The length of the pecimens is ranging from $5 \mathrm{~mm}$ to $80 \mathrm{~mm}$. The element size is $1 \mathrm{~mm}$, which is the same scale of the grain size of ice. Parameters used are the same as the cylindrical specimen above.

5 groups of specimens with different size are studied. The length of the specimens includes $5 \mathrm{~mm}, 10 \mathrm{~mm}, 20 \mathrm{~mm}$, $40 \mathrm{~mm}$ and $80 \mathrm{~mm}$ respectively. Under uniaxial compression, the average peak stress will increase when the size of the specimen decreases, as shown in Fig. 16. If the size is large enough, i.e. above $20 \mathrm{~mm}$ in this study, the average peak stress is independent of the specimen size. This is consistent with the result of experimental tests [18].

The strain rate effect is further studied with specimen size of $80 \mathrm{~mm}$. The test results and experimental results $[11,16,17,19,23,36,37]$ are in good agreement. At relatively low strain rate, i.e. lower than $10^{-3} \mathrm{~s}^{-1}$, the failure stress will increase when the strain rate decrease. When the strain rate is above $10^{-3} \mathrm{~s}^{-1}$, the failure stress will decrease with the increase of the strain rate. The strain rate effect in tension is also included in Fig. 17, and the numerical results is in accord with the results of Lee [23]. Comparing to compression, the variation range of failure stress in tension is much narrower. The difference is caused by different failure modes. The compressive strength is depending upon the compressive stress. When the strain rate is high, the stress increases fast and the strength also increases fast. Meanwhile, on the micro-crack surface, friction takes place. And the friction is also dependent of the compressive stress. This will help to resist further fracture of the material. So the compressive strength increases fast with the 1strain rate. However, the same as the tensile failure, if the strain rate is too high, local fracture close to the loading surface will take place. Change of failure modes finally results in the decrease of the compressive strength. 


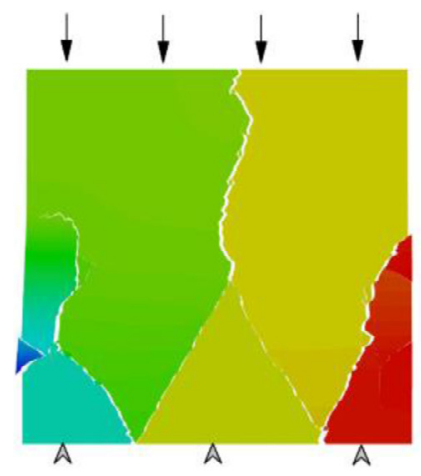

(a) Uniaxial loading

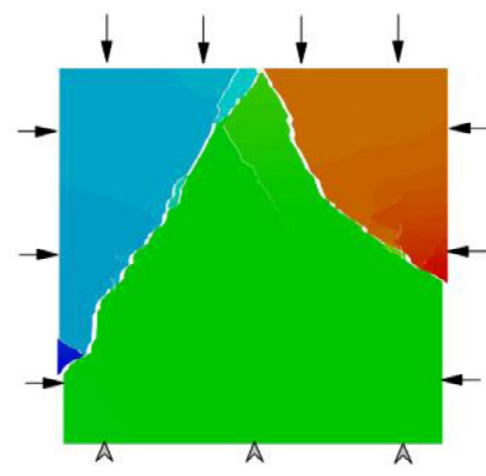

(b) Biaxial loading

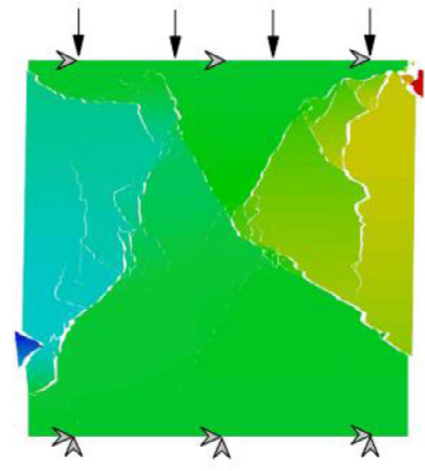

(c) End constrain

Fig. 18. Different failure modes under different boundary conditions. Under uniaxial loading, the failure mode is a combination of shear fault and longitudinal splitting. While under biaxial loading or compression with confinement, the principal failure mode is inclined shear fault. However, when the ends of the specimen are constrained, the failure mode is shear extrusion in the middle of the specimen.

Loading condition and boundary condition will also affect the failure modes, as shown in Fig. 18. Under uniaxial compression, the failure mode is a combination of shear fault and longitudinal splitting. However, with confinement, inclined shear failure plays a dominant role, since the confinement will prevent the longitudinal splitting. If the horizontal freedom at both ends of the specimen is constrained, which is corresponding to the extreme condition of coarse loading platens, the failure mode will exhibit shear extrusion in the middle of the specimen.

\section{Conclusions}

In order to simulate the brittle failure process of ice, a new numerical method, particle-subdomain method (PSM) is introduced at the first part of this paper. PSM is a dynamic explicit algorithm which has combined the advantages of material point method, dynamic relaxation method, finite element method (or other continuous element model) and discrete element method. The system of PSM consists of material particles and associate subdomains. At the very beginning, an initial mesh for the computational domain needs to be generated to establish the system. The material particles are formed by the nodes. Each particle has got a subdomain constructed by all the associate elements. The dynamic equation for the particle within a subdomain has been established with the energy function and Lagrange equation. Internal force of each subdomain applied to the associate particle can be calculated with finite element method. However, only element stiffness matrix is needed instead of the global stiffness matrix. Dynamic relaxation method is used to iterate over all the particles and subdomains to obtain the whole field result during each time step. The particles and subdomains could be divided into new ones in the calculation process when exceeding the strength. Thus, the cracks and new boundaries can be explicitly generated along the interface of the elements. Interactions at the cracks and boundaries as well as the collision of blocks are calculated with discrete element method. Numerical examples for wave propagation, brittle fracture, contact and motion have validated its effectiveness in problems of dynamic and fracture.

Mechanical properties for tensile and compressive failure of ice in laboratory scale are investigated with PSM. The heterogeneous and micro-discontinuous characteristics of ice are model with the distribution of strength. The failure process can be well described with PSM. The specimen shows four stages during the fracture process. At the very beginning, the ice material is continuous with linear elastic deformation. Then comes the nonlinear stage with homogeneous micro-cracks within the material until reaching the peak stress. Principal cracks start to nucleate at the peak stress and this is the signal for the third stage with strain softening. During this stage, localization bands are formed and the fractures would focus upon these bands until the last stage when global failure takes place.

It is also very interesting that the size effect and strain rate effect can be obtained and explained through the internal fracture process of the material. When the specimen size is too small, i.e. less than 10 times of grain size, the diversity of micro-structure and distributed character of the strength within the material is not so continuous, which has prevented the chain reaction of the progressive failure process. Through increase of the specimen size, the strength will quickly tend to a constant value. The transition property of strength due to strain rate are discussed within the range of $10^{-8}-10^{-2} \mathrm{~s}^{-1}$. Change of the strain rate will change the fracture state within the material and finally result in the variation of failure strength. Under low strain rate, the whole process can be regarded as a quasi-static problem, that the state of the computational field is equilibrium. Under this circumstance, crack nucleation will occur at the weakest part of the specimen and starts the progressive failure process. However, with the increase of the strain rate, the equilibrium state within the material would be disturbed. Fractures and localization bands will gradually move close to the loading surface, because the average stress close to the loading end will get larger than that in the still end with the increase of the strain rate. Crack nucleation and localization band may no longer occur at the weakest part, which leads to the increase of the tensile strength 
with increase of the strain rate in a certain range. This is also true for the compressive test. And moreover, the compressive strength depends upon the compressive stress. Increase of the loading rate will rapidly increase the strength. Even at the cracks, the friction will also increase with compressive stress, which will further prevent the development of new cracks. This is why the strain rate effect is much more remarkable in compression than in tension. But if the strain rate is higher than a certain value, the failure strength will exhibit a tendency of decrease with increase of the strain rate. This is because of the occurrence of local failures close to loading surface at the very beginning. Breakage and extrusion of material due to local fracture have blocked the propagation of stress to the far end and intensive local fractures dissipate lots of the energy, which results in the decrease of global strength.

Boundary conditions can affect the failure modes in compression. Without confinement, the specimen will show longitudinal splitting. However, inclined shear fault should be a dominant form in biaxial loading or with confinement. Constrain of loading ends will result in shear extrusion in the middle of the specimen.

The application of PSM in brittle failure of ice has further demonstrated its effectiveness in problems of dynamic and fracture. Many more attempts in other fields could be conducted with this new numerical method. Thus future work may extend the application of PSM in other materials, such as rock, glass or even metal. More theoretical and computational models should be developed to further improve this method, for example, failure model for different materials, element breakage mode to insure more fracture path and new contact model to further increase the computational efficiency and precision.

\section{Declaration of Competing Interest}

The authors declare that they have no known competing financial interests or personal relationships that could have appeared to influence the work reported in this paper.

The authors declare the following financial interests/personal relationships which may be considered as potential competing interests.

\section{CRediT authorship contribution statement}

Jian-Ping Zhang: Data curation, Formal analysis, Investigation, Resources, Validation, Visualization, Writing - review \& editing. Dong Zhou: Conceptualization, Data curation, Funding acquisition, Formal analysis, Methodology, Project administration, Resources, Software, Supervision, Validation, Writing - original draft, Writing - review \& editing.

\section{Acknowledgments}

The authors would like to acknowledge the financial support of China National Program on Key Basic Research Project (973 Program, Grant No. 2015CB250903) and the Strategic Priority Research Program (B) of Chinese Academy of Sciences (Grant No. XDB10030303).

\section{References}

[1] Brackbill JU. The ringing instability in particle-in-cell calculations of low-speed flow. J Comput Phys 1988;75(2):469-92

[2] Brackbill JU, Ruppel HM. FLIP: a method for adaptively zoned, particle-in-cell calculations of fluid flows in two dimensions. J Comput Phys 1986;65(2):314-43.

[3] Burgess D, Sulsky D, Brackbill JU. Mass matrix formulation of the FLIP particle-in-cell method. J Comput Phys 1992;103(1):1-15.

[4] Chu ML, Xian X, Scavuzzo RJ. In: Proceedings of 4th international workshop on atmospheric icing of structures, (EDF), Paris; 1988. p. 255-61.

[5] Cole DM. Grain size and the compressive strength of ice. J Energy Resour Technol 1985;107(3):369-74.

[6] Cole DM. Strain-rate and grain-size effects in ice. J Glaciol 1987;33(115):274-80.

[7] Coulomb CA. Sur une application des regles maximis et minimis a quelques problems de statique, relatives a l'architecture. Acad Sci Paris Mem Math Phys $1776 ; 7: 343-82$.

[8] Nadai A. Theory of flow and fracture of solids. New York: McGraw Hill; 1950.

[9] Dang HK, Meguid MA. Evaluating the performance of an explicit dynamic relaxation technique in analyzing non-linear geotechnical engineering problems. Comp Geotec 2010;37(1):125-31.

[10] Day AS. An introduction to dynamic relaxation (Dynamic relaxation method for structural analysis, using computer to calculate internal forces following development from initially unloaded state). Engineer 1965;219:218-21.

[11] Druez J, Nguyen DD, Lavoie Y. Mechanical properties of atmospheric ice. Cold Reg Sci Tech 1986;13(1):67-74

[12] Felippa CA. Dynamic relaxation and Quasi-Newton methods. Numerical methods for nonlinear problems 2. Swansea, UK: Pineridge Press; 1984. p. 27-38.

[13] Frederking R, Barker A. Friction of sea ice on various construction materials. In: Proceeding of the 16 th IAHR international symposium on ice, 1 ; 2002. p. $442-9$.

[14] Han SE, Lee KS. A study of the stabilizing process of unstable structures by dynamic relaxation method. Comput Struct 2003;81(17):1677-88.

[15] Harlow FH. The particle-in-cell computing method for fluid dynamics. Meth Comput Phys 1964;3:319-43.

[16] Hawkes I, Mellor M. Deformation and fracture of ice under uniaxial stress. J Glaciol 1972;11(61):103-31.

[17] Jones SJ. The confined compressive strength of polycrystalline ice. J Glaciol 1982;28(98):171-7.

[18] Jones SJ, Chew HAM. Effect of sample and grain size on the compressive strength of ice. Ann Glaciol 1983;4:129-32

[19] Kermani M, Farzaneh M, Gagnon R. Compressive strength of atmospheric ice. Cold Reg Sci Tech 2007;49(3):195-205.

[20] Kuehn GA, Lee RW, Nixon WA, et al. . The structure and tensile behavior of first-year sea ice and laboratory grown saline ice. J Offshore Mech Arctic Eng 1990;112:357-63.

[21] Kuehn GA, Schulson EM, Jones DE, et al. . The compressive strengths of ice cubes of different sizes. J Offshore Mech Arctic Eng. 1993;115(2):142-8.

[22] Labuz J, Zang A. Mohr-coulomb failure criterion. Rock Mech. Rock Eng 2012;45:975-9. 
[23] Lee RW. The effect of grain size on the tensile strength of ice at two strain-rates PhD Thesis. Thayer School of Engineering; 1985.

[24] Li SH, Zhou D. Progressive failure constitutive model of fracture plane in geomaterial based on strain strength distribution. Int J Solids Struct 2013;50(3-4):570-7.

[25] Mohamed AMA, Farzaneh M. An experimental study on the tensile properties of atmospheric ice. Cold Reg Sci Tech 2011:68(3):91-8.

[26] Mohr O. Welche Umstände bedingen die Elastizitätsgrenze und den Bruch eines Materials? Zeit des Ver Deut Ing 1900;44:1524-30.

[27] Moslet PO. Field testing of uniaxial compression strength of columnar sea ice. Cold Reg Sci Tech 2007;48(1):1-14.

[28] Murat JR, Lainey LM. Some experimental observations on the Poisson's ratio of sea-ice. Cold Reg Sci Tech 1982;6(2):105-13.

[29] Oakley DR, Knight NF Jr. Adaptive dynamic relaxation algorithm for non-linear hyperelastic structures part I. Formulation. Comput Meth Appl Mech Eng 1995;126(1):67-89.

[30] Paul B. Generalized pyramidal fracture and yield criteria. Int J Solids Struct 1968;4:175-96.

[31] Pernas-Sánchez J, Pedroche DA, Varas D, et al. Numerical modeling of ice behavior under high velocity impacts. Int J Solids Struct 2012;49(14):1919-27.

[32] Petrovic JJ. Review mechanical properties of ice and snow. J Mater Sci 2003;38(1):1-6.

[33] Read HE, Hegemier GA. Strain softening of rock, soil and concrete - a review article. Mech Mater 1984;3(4):271-94.

[34] Richter-Menge JA, Jones KF. The tensile strength of first-year sea ice. J Glaciol 1993;39(133):609-18.

[35] Sammonds PR, Murrell SAF, Rist MA. Fracture of multiyear sea ice. J Geophys Res 1998;103(C10):21795-815.

[36] Schulson EM, Canon NP. The effect of grain size on the compressive strength of ice. In: IAHR ice symposium, Hamburg; 1984. p. 109-17.

[37] Schulson EM. The brittle compressive fracture of ice. Acta Metall Mater 1990;38(10):1963-76.

[38] Schulson EM. Brittle failure of ice. Eng Fract Mech 2001;68(17):1839-87.

[39] Shazly M, Prakash V, Lerch BA. High strain-rate behavior of ice under uniaxial compression. Int J Solids Struct 2009;46(6):1499-515.

[40] Timco GW, Frederking RMW. Confined compression tests: outlining the failure envelope of columnar sea ice. Cold Reg Sci Tech 1986;12(1):13-28.

[41] Timco GW, Frederking RMW. Compressive strength of sea ice sheets. Cold Reg Sci Tech 1990;17(3):227-40.

[42] Timco GW, Weeks WF. A review of the engineering properties of sea ice. Cold Reg Sci Tech 2010;60(2):107-29.

[43] Tippmann JD, Kim H, Rhymer JD. Experimentally validated strain rate dependent material model for spherical ice impact simulation. Int J Impact Eng 2013;57:43-54

[44] Underwood PG. Dynamic relaxation: a review. Computational methods for transient dynamic analysis. Belytschko T, Hughes TJR, editors. editors, Amsterdam: North Holland; 1983. [chapter 5].

[45] Wang Q Li Z, Lei R, Lu P, Han H. Estimation of the uniaxial compressive strength of arctic sea ice during melt season. Cold Reg Sci Tech 2018;151:9-18.

[46] Weibull W, Stockholm S. A statistical distribution function of wide application. J Appl Mech 1951;18:293-7.

[47] Xu Y, Xu J, Wang J. Fractal model for size effect on ice failure strength. Cold Reg Sci Tech 2004;40(1):135-44

[48] Yang B, Zhang G, Huang Z, Sun Z, Zong Z. Numerical simulation of the ice resistance in pack ice conditions. Int J Comput Meth 2018:1844005.

[49] Zhang S, El Kerdi O, Khurram RA, Habashi WG. FEM analysis of in-flight ice break-up. Finite Elem Anal Des 2012;57:55-66. 